\title{
First-principles method for impurities in quantum fluids: Positron in an electron gas
}

\author{
N. D. Drummond, P. López Ríos, C. J. Pickard, * and R. J. Needs \\ TCM Group, Cavendish Laboratory, University of Cambridge, JJ Thomson Avenue, Cambridge CB3 OHE, United Kingdom \\ (Received 25 February 2010; revised manuscript received 18 June 2010; published 9 July 2010)
}

\begin{abstract}
We propose a first-principles methodology for calculating the behavior of isolated impurities immersed in quantum fluids. To obtain an accurate description of correlation effects between the impurity and the host, we work in the frame of reference in which the impurity is stationary, building on the work of C. H. Leung, M. J. Stott, and C. O. Almbladh [Phys. Lett. 57A, 26 (1976)]. We apply our methodology to the case of a positron immersed in an electron gas. Our positron relaxation energies and annihilation rates are similar to those from the best existing many-body calculations. Our annihilating-pair momentum densities are significantly different from previous data and include a "tail" after the Fermi edge.
\end{abstract}

DOI: 10.1103/PhysRevB.82.035107

PACS number(s): 78.70.Bj, 71.10.Ca, 71.15.Mb, 71.60.+z

\section{INTRODUCTION}

Quantum impurity problems are of widespread interest in condensed-matter physics. Here we study a positron in a homogeneous electron gas (HEG) as an example of an impurity problem in which a particle of one species is immersed in a fluid of another species, and where quantum effects are important for both the host and impurity. Examples of other problems of this type are the Mahan exciton ${ }^{1}$ in semiconductor physics, in which a hole in the valence band interacts with an electron gas in the conduction band (or an electron interacts with a hole gas), and an impurity atom in a BoseEinstein condensate. Our approach requires knowledge of the explicit interaction between the host and impurity particles and the availability of a reasonably accurate mean-field description of the host, such as the Kohn-Sham densityfunctional theory (DFT) (Refs. 2 and 3) of electrons or the Gross-Pitaevskii equation ${ }^{4,5}$ for Bose-Einstein condensates. An important characteristic of our approach is the inclusion of nonadiabatic impurity-host effects. We have chosen to present results for the positron problem, firstly, because they can be compared with data in the literature to gauge their accuracy and, secondly, because nonadiabatic effects are expected to be important as the electron and positron are of equal mass. Applications of our method to other problems such as those mentioned above require only straightforward modifications.

There are several experimental techniques for studying defects in materials that involve injecting small numbers of positrons into samples and measuring the resulting annihilation radiation. ${ }^{6}$ On entering condensed matter, a low-energy positron thermalizes rapidly and may be trapped by openvolume defects such as vacancies, where the nuclear repulsion is weak. The positron lifetime is measured as the time interval between the detection of a photon emitted in the $\beta^{+}$ radioactive decay that produces the positron and the detection of the two $0.511 \mathrm{MeV}$ photons emitted when the positron annihilates an opposite-spin electron. ${ }^{6}$ The lifetime is characteristic of the defect at which the positron settles, and positron annihilation spectroscopy is an important, nondestructive technique for characterizing open-volume defects. Measuring the Doppler broadening of the annihilation radiation or the angular correlation between the two $0.511 \mathrm{MeV}$ photons yields information about the momentum density (MD) of the electrons in the presence of the positron. Modeling is then required to extract information about the unperturbed system from the experimental data.

This paper is arranged as follows. In Sec. II we discuss the use of two-component DFT in studies of positrons in real materials. In Sec. III we describe our methodology for treating quantum impurities. In Sec. IV we discuss the calculation of the relaxation energy. In Sec. V we discuss the calculation of the annihilating-pair MD and in Sec. VI we discuss the calculation of the electron-positron pair-correlation function (PCF) and annihilation rate. We describe the elimination of finite-basis and finite-size errors from our results in Sec. VII. We present our results in Sec. VIII. Finally, we draw our conclusions in Sec. IX.

Throughout we use Hartree atomic units, in which the Dirac constant, the electronic mass, the magnitude of the electronic charge, and $4 \pi$ times the permittivity of free space are unity: $\hbar=m_{e}=|e|=4 \pi \epsilon_{0}=1$. We specify the density of a HEG by the radius $r_{s}$ of the sphere that contains one electron on average in units of the Bohr radius.

\section{TWO-COMPONENT DFT FOR POSITRONS IN REAL MATERIALS}

The most widely used method for modeling positrons in real materials is two-component $\mathrm{DFT}^{7}$ in which the interaction between the electrons and the positron is described by a functional of the densities of the electron and positron components. Within the local-density approximation (LDA), this functional is obtained directly from the relaxation energy $\Delta \Omega$ of a positron in a HEG, which is the difference between the energy of a HEG with and without a positron immersed in it. Equivalently, $\Delta \Omega$ is the electron-positron correlation energy.

Two-component DFT can lead to accurate electron and positron densities but the DFT orbitals yield very poor annihilating-pair MDs $\rho(\bar{p})$ and electron-positron PCFs $g(r)$ because they do not include the full effects of the strong electron-positron correlation. ${ }^{7,8}$ The results can, however, be substantially improved by correcting the calculated PCFs and MDs using accurate data for a positron in a HEG., ${ }^{7,8}$ The effective electron density felt by the positron, and hence the annihilation rate, is proportional to the contact PCF between 
the positron and the electrons, $g(0)$. The annihilation rate for a positron immersed in a paramagnetic HEG is $\lambda$ $=3 g(0) /\left(4 c^{3} r_{s}^{3}\right)$, where $r_{s}$ is the electron density parameter and $c$ is the speed of light in vacuo. ${ }^{6}$ If the electron and positron motions were uncorrelated then $g(0)$ would be unity, but the strong Coulomb attraction leads to much larger values, particularly at low densities, where a bound state may be formed. Together, our results for $\Delta \Omega, g(0)$, and $\rho(\bar{p})$ permit the construction of a two-component DFT within the LDA for a positron in a HEG, which in turn will enable the calculation of the annihilation rates and MDs used to interpret the results of positron annihilation experiments.

\section{GENERATING ORBITALS FOR AN IMPURITY-IN-HEG SYSTEM}

\section{A. Hamiltonian}

Consider a single impurity particle of mass $m_{p}$ and charge $q_{p}$ immersed in a gas of electrons in a finite cell subject to periodic boundary conditions. Suppose the electron mass is $m_{e}$ and the electron charge is $q_{e}$. Let the electron coordinates be $\left\{\mathbf{r}_{i}\right\}$ and the impurity position be $\mathbf{s}$ and define nondimensional coordinates $\widetilde{\mathbf{r}}_{i}=\mathbf{r} / r_{s}$ and $\widetilde{\mathbf{s}}=\mathbf{S} / r_{s}$. In terms of these coordinates the boundary conditions on the wave function are independent of $r_{s}$, and the $r_{s}$ dependence appears explicitly in the Hamiltonian, which is

$$
\begin{aligned}
\hat{H}\left(r_{s}, m_{e}, q_{e}, m_{p}, q_{p}\right)= & \sum_{i=1}^{N} \frac{-1}{2 m_{e} r_{s}^{2}} \nabla_{\widetilde{\mathbf{r}}_{i}}^{2}-\frac{1}{2 m_{p} r_{s}^{2}} \nabla_{\widetilde{\mathbf{s}}}^{2} \\
& +\sum_{i=1}^{N} q_{e} q_{p} v_{E}\left[\left(\widetilde{\mathbf{r}}_{i}-\widetilde{\mathbf{s}}\right) r_{s}\right] \\
& +\sum_{i=1}^{N-1} \sum_{j=i+1}^{N} q_{e}^{2} v_{E}\left[\left(\widetilde{\mathbf{r}}_{i}-\widetilde{\mathbf{r}}_{j}\right) r_{s}\right] \\
& +\frac{\left(N+q_{p}^{2}\right) v_{M}\left(r_{s}\right)}{2},
\end{aligned}
$$

where $v_{E}$ is the Ewald interaction and $v_{M}$ is the Madelung constant. Note that $v_{M}\left(r_{s}\right)=v_{M}(1) / r_{s}$ and $v_{E}\left(\widetilde{\mathbf{r}} r_{s}\right)=v_{E}(\widetilde{\mathbf{r}}) / r_{s}$. It is therefore easy to show that

$$
\hat{H}\left(r_{s}, m_{e}, q_{e}, m_{p}, q_{p}\right)=\hat{H}\left(m_{e} q_{e}^{2} r_{s}, 1,-1, m_{p} / m_{e},-q_{p} / q_{e}\right) m_{e} q_{e}^{4} .
$$

Henceforth we shall assume that $m_{e}=1$ a.u. and $q_{e}=$ -1 a.u. since results for other electron masses can be obtained by rescaling the density and energy.

\section{B. Coordinate transformation}

\section{Transformation of the Hamiltonian}

We follow the suggestion of Leung et al. ${ }^{9}$ that it is useful to describe a positron in a HEG using the set of electron positions relative to the position of the positron. The advantage of this formulation is that the mean-field singledeterminant approximation for the wave function of this system includes explicit electron-positron correlation, whereas in the laboratory frame the ground state consists of a completely delocalized positron and a HEG.

Throughout this section we write $\nabla_{i} f$ as $\partial f / \partial \mathbf{r}_{i}, \nabla_{i}^{2} f$ as $\partial^{2} f / \partial \mathbf{r}_{i}^{2}$, etc. The Hamiltonian can be written as

$$
\begin{aligned}
\hat{H}= & \sum_{i=1}^{N} \frac{-1}{2} \frac{\partial^{2}}{\partial \mathbf{r}_{i}^{2}}-\frac{1}{2 m_{p}} \frac{\partial^{2}}{\partial \mathbf{s}^{2}}-\sum_{i=1}^{N} q_{p} v_{E}\left(\mathbf{r}_{i}-\mathbf{s}\right) \\
& +\sum_{i=1}^{N-1} \sum_{j=i+1}^{N} v_{E}\left(\mathbf{r}_{i}-\mathbf{r}_{j}\right)+\frac{\left(N+q_{p}^{2}\right) v_{M}}{2} .
\end{aligned}
$$

Let

$$
\begin{array}{r}
\mathbf{X}=\frac{1}{N+m_{p}}\left(\sum_{i=1}^{N} \mathbf{r}_{i}+m_{p} \mathbf{s}\right) \text { and } \\
\mathbf{x}_{i}=\mathbf{r}_{i}-\mathbf{s} \quad \forall i \in\{1, \ldots, N\} .
\end{array}
$$

Then it can be shown that

$$
\begin{gathered}
\frac{\partial^{2}}{\partial \mathbf{s}^{2}}=\sum_{i=1}^{N} \sum_{j=1}^{N} \frac{\partial^{2}}{\partial \mathbf{x}_{i} \partial \mathbf{x}_{j}}-\frac{2 m_{p}}{N+m_{p}} \sum_{j=1}^{N} \frac{\partial^{2}}{\partial \mathbf{X} \partial \mathbf{x}_{j}}+\left(\frac{m_{p}}{N+m_{p}}\right)^{2} \frac{\partial^{2}}{\partial \mathbf{X}^{2}}, \\
\frac{\partial^{2}}{\partial \mathbf{r}_{i}^{2}}=\frac{\partial^{2}}{\partial \mathbf{x}_{i}^{2}}+\frac{2}{N+m_{p}} \frac{\partial^{2}}{\partial \mathbf{X} \partial \mathbf{x}_{i}}+\left(\frac{1}{N+m_{p}}\right)^{2} \frac{\partial^{2}}{\partial \mathbf{X}^{2}}
\end{gathered}
$$

Hence

$$
\begin{aligned}
\hat{H}= & -\frac{1}{2\left(N+m_{p}\right)} \frac{\partial^{2}}{\partial \mathbf{X}^{2}}+\left\{\sum_{i=1}^{N}\left[-\frac{1}{2}\left(1+\frac{1}{m_{p}}\right) \frac{\partial^{2}}{\partial \mathbf{x}_{i}^{2}}-q_{p} v_{E}\left(\mathbf{x}_{i}\right)\right]\right. \\
& \left.+\sum_{i=1}^{N-1} \sum_{j=i+1}^{N}\left[-\frac{1}{m_{p}} \frac{\partial^{2}}{\partial \mathbf{x}_{i} \partial \mathbf{x}_{j}}+v_{E}\left(\mathbf{x}_{i}-\mathbf{x}_{j}\right)\right]+\frac{\left(N+q_{p}^{2}\right) v_{M}}{2}\right\} \\
\equiv & \hat{T}_{\mathrm{CM}}+\hat{H}^{\prime}
\end{aligned}
$$

where $\hat{T}_{\mathrm{CM}}$ is the center-of-mass kinetic-energy operator and $\hat{H}^{\prime}$ is the Hamiltonian for $N$ interacting particles of mass $\mu$ $\equiv m_{p} /\left(1+m_{p}\right)$ a.u. and charge -1 a.u. in the presence of a fixed charge of magnitude $q_{p}$ a.u. at the origin. In addition to the usual Ewald interaction between particles there is an interaction term $-\left(1 / m_{p}\right) \sum_{i=1}^{N-1} \sum_{j=i+1}^{N}\left(\partial / \partial \mathbf{x}_{i}\right) \cdot\left(\partial / \partial \mathbf{x}_{j}\right)$.

In order to reduce finite-size effects we may use $\mathbf{k}$-point sampling within one-electron theories. Physically, this means creating a simulation cell containing multiple copies of a primitive cell, each of which contains a single impurity. The many-electron Hamiltonian is 


$$
\begin{aligned}
\hat{H}^{\prime}= & \sum_{i=1}^{N}\left[-\frac{1}{2 \mu} \frac{\partial^{2}}{\partial \mathbf{x}_{i}^{2}}-\sum_{\mathbf{R}_{p} \in V_{s}} q_{p} v_{E}\left(\mathbf{x}_{i}-\mathbf{R}_{p}\right)\right] \\
& +\sum_{i=1}^{N-1} \sum_{j=i+1}^{N}\left[-\frac{1}{m_{p}} \frac{\partial^{2}}{\partial \mathbf{x}_{i} \partial \mathbf{x}_{j}}+v_{E}\left(\mathbf{x}_{i}-\mathbf{x}_{j}\right)\right] \\
& +\frac{\left(N+N_{p}^{4 / 3} q_{p}^{2}\right) v_{M}}{2},
\end{aligned}
$$

where the $\left\{\mathbf{R}_{p}\right\}$ are the primitive-cell lattice vectors, $V_{s}$ is the simulation-cell volume, $N$ is the number of electrons in the simulation cell, and $N_{p}$ is the number of primitive cells in the simulation cell. For a given primitive cell, increasing the k-point sampling increases the size of the simulation cell and hence reduces single-particle finite-size effects. Given finite computer resources, it may be preferable to use $\mathbf{k}$-point sampling rather than simply increasing the size of the primitive cell, because the number of orbital coefficients grows linearly with the number of $\mathbf{k}$ points but quadratically with the size of the primitive cell. However, if multiple $\mathbf{k}$ points are used then $\hat{H}^{\prime}$ no longer corresponds exactly to an untransformed Hamiltonian for a single impurity in a periodic cell. Furthermore, k-point sampling does not reduce the finite-size error due to the interaction of images of the impurity particle.

\section{Boundary conditions on the transformed wave function}

The wave function $\Psi\left(\mathbf{s} ; \mathbf{r}_{1}, \ldots, \mathbf{r}_{N}\right)$ is antisymmetric with respect to the exchange of $\mathbf{r}_{i}$ and $\mathbf{r}_{j}$ if and only if $\Psi\left(\mathbf{X} ; \mathbf{x}_{1}, \ldots, \mathbf{x}_{N}\right)$ is antisymmetric with respect to exchange of $\mathbf{x}_{i}$ and $\mathbf{x}_{j}$, so the coordinate transformation preserves the antisymmetry of the wave function with respect to same-spin electrons.

The wave function must satisfy the many-body Bloch theorem, ${ }^{10,11}$

$$
\Psi\left(\mathbf{s} ; \mathbf{r}_{1}, \ldots, \mathbf{r}_{N}\right)=U_{\mathbf{k}_{s}}\left(\mathbf{s} ; \mathbf{r}_{1}, \ldots, \mathbf{r}_{N}\right) \exp \left[i \mathbf{k}_{s} \cdot\left(\mathbf{s}+\sum_{i=1}^{N} \mathbf{r}_{i}\right)\right]
$$

where the Bloch vector $\mathbf{k}_{s}$ lies in the first Brillouin zone of the simulation cell and $U$ is invariant under the translation of $\mathbf{S}$ or any of the $\left\{\mathbf{r}_{i}\right\}$ through a simulation-cell lattice vector $\mathbf{R}_{s}$. Furthermore, the Hamiltonian is separable in our transformed coordinates [see Eq. (7)], so we may write

$$
\Psi\left(\mathbf{s} ; \mathbf{r}_{1}, \ldots, \mathbf{r}_{N}\right)=\Phi_{\mathbf{P}}\left(\mathbf{x}_{1}, \ldots, \mathbf{x}_{N}\right) \exp (i \mathbf{P} \cdot \mathbf{X}),
$$

where $\mathbf{P}$ is the center-of-mass momentum, which can take any value consistent with the choice of Bloch vector [see Eq. (12)]. In order to specify fully the boundary conditions on the wave function, we need to choose the values of both $\mathbf{k}_{s}$ and $\mathbf{P} .^{10,11}$ Our untransformed system is homogeneous and therefore invariant under the translation of all particles through any vector whatsoever. Hence $\mathbf{P}$ is not constrained to lie in the first Brillouin zone of the primitive cell, as is the case for an inhomogeneous periodic system. ${ }^{10,11}$
For all simulation-cell lattice vectors $\mathbf{R}_{s}$ and impurity particle and electron coordinates,

$$
\begin{aligned}
\Psi & \left(\mathbf{s} ; \mathbf{r}_{1}, \ldots, \mathbf{r}_{N}\right) \exp \left[i \mathbf{k}_{s} \cdot(N+1) \mathbf{R}_{s}\right] \\
& =\Psi\left(\mathbf{s}+\mathbf{R}_{s} ; \mathbf{r}_{1}+\mathbf{R}_{s}, \ldots, \mathbf{r}_{N}+\mathbf{R}_{s}\right) \\
& =\Phi_{\mathbf{P}}\left(\mathbf{x}_{1}, \ldots, \mathbf{x}_{N}\right) \exp \left[i \mathbf{P} \cdot\left(\mathbf{X}+\mathbf{R}_{s}\right)\right] \\
& =\Psi\left(\mathbf{s} ; \mathbf{r}_{1}, \ldots, \mathbf{r}_{N}\right) \exp \left(i \mathbf{P} \cdot \mathbf{R}_{s}\right)
\end{aligned}
$$

Hence

$$
\mathbf{P}=(N+1) \mathbf{k}_{s}-\mathbf{G}_{s}^{\mathrm{CM}},
$$

where $\mathbf{G}_{s}^{\mathrm{CM}}$ is a simulation-cell reciprocal-lattice vector.

Now consider translating $\mathbf{r}_{1}$ through a simulation-cell lattice vector $\mathbf{R}_{s}$, keeping the other coordinates fixed. In that case $\mathbf{x}_{1}$ is mapped to $\mathbf{x}_{1}+\mathbf{R}_{s}$ and $\mathbf{X}$ is mapped to $\mathbf{X}+\mathbf{R}_{s} /(N$ $\left.+m_{p}\right)$. It follows from Eqs. (9) and (10) that

$$
\begin{aligned}
\Phi_{\mathbf{P}}\left(\mathbf{x}_{1}\right. & \left.+\mathbf{R}_{s}, \mathbf{x}_{2}, \ldots, \mathbf{x}_{N}\right) \\
= & \Phi_{\mathbf{P}}\left(\mathbf{x}_{1}, \ldots, \mathbf{x}_{N}\right) \\
& \quad \times \exp \left[i\left(\mathbf{k}_{s}-\frac{1}{N+m_{p}} \mathbf{P}\right) \cdot \mathbf{R}_{s}\right] .
\end{aligned}
$$

A similar result is obtained when the other electrons are translated. So the wave function $\Phi_{\mathbf{P}}\left(\mathbf{x}_{1}, \ldots, \mathbf{x}_{N}\right)$ satisfies twisted boundary conditions with a Bloch vector

$$
\mathbf{k}_{s}^{\prime}=\mathbf{k}_{s}-\frac{1}{N+m_{p}} \mathbf{P}
$$

The center-of-mass kinetic energy is

$$
T_{\mathrm{CM}}=\frac{|\mathbf{P}|^{2}}{2\left(N+m_{p}\right)} \text {. }
$$

This must be added to the ground-state energy of the system described by $\hat{H}^{\prime}$ to obtain the total ground-state energy. In the ground state for a given $\mathbf{k}_{s}$ one should choose $\mathbf{G}_{s}^{\mathrm{CM}}$ so that $T_{\mathrm{CM}}$ is minimized, in which case $\mathbf{P}$ is simply equal to $(N+1) \mathbf{k}_{s}$ reduced into the first Brillouin zone of the simulation cell.

As discussed in Sec. III C 1, the method used to evaluate the extra terms requires $\mathbf{k}_{s}^{\prime}=\mathbf{G}_{s} / 2$ for some simulation-cell reciprocal-lattice vector $\mathbf{G}_{s}$. By far the easiest way to satisfy the two conditions on $\mathbf{k}_{s}^{\prime}$ is to choose $\mathbf{k}_{s}=\mathbf{P}=\mathbf{0}$, in which case $\mathbf{k}_{s}^{\prime}=\mathbf{0}$ and $T_{\mathrm{CM}}=0$. When simulating the system described by $\hat{H}^{\prime}$, the $\Gamma$ point should therefore be included in the $\mathbf{k}$-point mesh. 
It might be imagined that violating the boundary conditions on the wave function required for an exact correspondence with an untransformed system would not matter. As already noted, the use of multiple $\mathbf{k}$ points always eliminates the possibility of correspondence with an untransformed system. In practice, however, it appears to be essential to satisfy the boundary conditions and to use just a single $\mathbf{k}$ point, as discussed in Sec. III E 2.

\section{Evaluating the extra interaction terms}

\section{Expectation value with respect to a Slater determinant of orthonormal orbitals}

Henceforth, $\nabla_{i} \equiv \partial / \partial \mathbf{x}_{i}$. Let

$$
D^{\uparrow}\left(\mathbf{x}_{1}, \ldots, \mathbf{x}_{N_{\uparrow}}\right)=\frac{1}{\sqrt{N_{\uparrow} !}}\left|\begin{array}{ccc}
\phi_{1}^{\uparrow}\left(\mathbf{x}_{1}\right) & \cdots & \phi_{N_{\uparrow}}^{\uparrow}\left(\mathbf{x}_{1}\right) \\
\vdots & & \vdots \\
\phi_{1}^{\uparrow}\left(\mathbf{x}_{N_{\uparrow}}\right) & \cdots & \phi_{N_{\uparrow}}^{\uparrow}\left(\mathbf{x}_{N_{\uparrow}}\right)
\end{array}\right|,
$$

where the orbitals are orthonormal, i.e., $\left\langle\phi_{i}^{\uparrow} \mid \phi_{j}^{\uparrow}\right\rangle=\delta_{i j} \forall i, j \in\left\{1, \ldots, N_{\uparrow}\right\}$. Then

$$
\left\langle D^{\uparrow} \mid \sum_{i=1}^{N_{\uparrow}-1} \sum_{j=i+1}^{N_{\uparrow}} \nabla_{i} \cdot \nabla_{j} D^{\uparrow}\right\rangle=\sum_{i=1}^{N_{\uparrow}-1} \sum_{j=i+1}^{N_{\uparrow}}\left(\left\langle\boldsymbol{\phi}_{i}^{\uparrow} \mid \nabla \boldsymbol{\phi}_{i}^{\uparrow}\right\rangle \cdot\left\langle\boldsymbol{\phi}_{j}^{\uparrow} \mid \nabla \boldsymbol{\phi}_{j}^{\uparrow}\right\rangle-\left\langle\boldsymbol{\phi}_{i}^{\uparrow} \mid \nabla \boldsymbol{\phi}_{j}^{\uparrow}\right\rangle \cdot\left\langle\boldsymbol{\phi}_{j}^{\uparrow} \mid \nabla \boldsymbol{\phi}_{i}^{\uparrow}\right\rangle\right) .
$$

Likewise,

$$
\left\langle D^{\uparrow} \mid \sum_{i=1}^{N_{\uparrow}} \nabla_{i} D^{\uparrow}\right\rangle=\sum_{i=1}^{N_{\uparrow}}\left\langle\phi_{i}^{\uparrow} \mid \nabla \phi_{i}^{\uparrow}\right\rangle
$$

Now let $\Phi\left(\mathbf{x}_{1}, \ldots, \mathbf{x}_{N}\right)=D^{\uparrow}\left(\mathbf{x}_{1}, \ldots, \mathbf{x}_{N_{\uparrow}}\right) D^{\downarrow}\left(\mathbf{x}_{N_{\uparrow}+1}, \ldots, \mathbf{x}_{N}\right)$, where $D^{\downarrow}$ is a Slater determinant of orbitals for spin-down electrons. Suppose $j>i$. Then

$$
\left\langle\Phi \mid \nabla_{i} \cdot \nabla_{j} \Phi\right\rangle= \begin{cases}\left\langle D^{\uparrow} \mid \nabla_{i} \cdot \nabla_{j} D^{\uparrow}\right\rangle & \text { if } i \text { and } j \text { are both spin up } \\ \left\langle D^{\downarrow} \mid \nabla_{i} \cdot \nabla_{j} D^{\downarrow}\right\rangle & \text { if } i \text { and } j \text { are both spin down } \\ \left\langle D^{\uparrow} \mid \nabla_{i} D^{\uparrow}\right\rangle \cdot\left\langle D^{\downarrow} \mid \nabla_{j} D^{\downarrow}\right\rangle & \text { if } i \text { and } j \text { have opposite spins. }\end{cases}
$$

Hence

$$
\begin{aligned}
\left\langle\Phi \mid \sum_{i=1}^{N-1} \sum_{j=i+1}^{N} \nabla_{i} \cdot \nabla_{j} \Phi\right\rangle & =\left\langle D^{\uparrow} \mid \sum_{i=1}^{N_{\uparrow}-1} \sum_{j=i+1}^{N_{\uparrow}} \nabla_{i} \cdot \nabla_{j} D^{\uparrow}\right\rangle+\left\langle D^{\uparrow} \mid \sum_{i=1}^{N_{\uparrow}} \nabla_{i} D^{\uparrow}\right\rangle \cdot\left\langle D^{\downarrow} \mid \sum_{j=N_{\uparrow}+1}^{N} \nabla_{j} D^{\downarrow}\right\rangle+\left\langle D^{\downarrow} \mid \sum_{i=N_{\uparrow}+1}^{N-1} \sum_{j=i+1}^{N} \nabla_{i} \cdot \nabla_{j} D^{\downarrow}\right\rangle \\
& =\frac{1}{2} \sum_{i, \alpha} \sum_{j, \beta}\left(\left\langle\phi_{i}^{\alpha} \mid \nabla \phi_{i}^{\alpha}\right\rangle \cdot\left\langle\phi_{j}^{\beta} \mid \nabla \phi_{j}^{\beta}\right\rangle-\delta_{\alpha \beta}\left\langle\phi_{i}^{\alpha} \mid \nabla \phi_{j}^{\beta}\right\rangle \cdot\left\langle\phi_{j}^{\beta} \mid \nabla \phi_{i}^{\alpha}\right\rangle\right),
\end{aligned}
$$

where we have made use of the fact that the terms with $\alpha$ $=\beta$ and $i=j$ in Eq. (19) cancel.

Suppose $\phi_{i}^{\alpha}$ is an eigenfunction of a single-particle Hamiltonian with eigenvalue $\mathcal{E}_{i}^{\alpha}$. Then, so long as it is compatible with the boundary conditions on the simulation cell, $\phi_{i}^{\alpha *}$ is also an eigenfunction with eigenvalue $\mathcal{E}_{i}^{\alpha}$, as can be seen by taking the complex conjugate of the single-particle Schrödinger equation. So, either $\phi_{i}^{\alpha *} \propto \phi_{i}^{\alpha}$ or $\phi_{i}^{\alpha *}$ and $\phi_{i}^{\alpha}$ are a complex-conjugate pair of degenerate eigenfunctions.

It is easy to show by integration by parts that $\left\langle\phi_{i}^{\alpha} \mid \nabla \phi_{i}^{\alpha}\right\rangle$ $+\left\langle\phi_{i}^{\alpha *} \mid \nabla \phi_{i}^{\alpha *}\right\rangle=\mathbf{0}$. If $\phi_{i}^{\alpha *} \propto \phi_{i}^{\alpha}$ then $\left\langle\phi_{i}^{\alpha} \mid \nabla \phi_{i}^{\alpha}\right\rangle=\mathbf{0}$. So, if all the occupied orbitals are complex-conjugate pairs or satisfy $\phi_{i}^{\alpha *} \propto \phi_{i}^{\alpha}$ then

$$
\sum_{i=1}^{N_{\alpha}}\left\langle\phi_{i}^{\alpha} \mid \nabla \phi_{i}^{\alpha}\right\rangle=\mathbf{0}
$$

and hence the direct term in Eq. (19) is zero.

The complex conjugate of a Bloch orbital with Bloch vector $\mathbf{k}$ is a Bloch orbital with Bloch vector $-\mathbf{k}$. Suppose we have chosen a particular simulation-cell Bloch vector $\mathbf{k}_{s}^{\prime}$. Then each single-particle Bloch vector must be of the form $\mathbf{k}=\mathbf{k}_{s}^{\prime}+\mathbf{G}_{s}$, where $\mathbf{G}_{s}$ is a reciprocal-lattice point of the simulation cell. It can be seen that $-\mathbf{k}$ is a legitimate Bloch vector if and only if the $\mathbf{k}_{s}^{\prime}=\mathbf{G}_{s} / 2$ for some $\mathbf{G}_{s}$. Hence it is only possible to occupy the orbitals in complex-conjugate pairs if $\mathbf{k}_{s}^{\prime}=\mathbf{G}_{s} / 2$ for some $\mathbf{G}_{s}$. 
In a closed-shell ground state (for which the conditions described above are satisfied) the expectation value of the extra interaction is

$$
\left\langle\Phi \mid-\frac{1}{m_{p}} \sum_{i=1}^{N-1} \sum_{j=i+1}^{N} \nabla_{i} \cdot \nabla_{j} \Phi\right\rangle=-\frac{1}{2 m_{p}} \sum_{\alpha} \sum_{i=1}^{N_{\alpha}} \sum_{j=1}^{N_{\alpha}}\left|\left\langle\phi_{i}^{\alpha} \mid \nabla \phi_{j}^{\alpha}\right\rangle\right|^{2},
$$

which is clearly negative.

Suppose

$$
\phi_{i}^{\alpha}(\mathbf{x})=\sum_{\mathbf{G}_{p}} c_{i \mathbf{G}_{p}}^{\alpha} \exp \left[i\left(\mathbf{k}_{i}^{\alpha}+\mathbf{G}_{p}\right) \cdot \mathbf{x}\right],
$$

where $V_{p}$ is the volume of the primitive unit cell, the $c_{i \mathbf{G}_{p}}^{\alpha}$ are plane-wave expansion coefficients, the $\left\{\mathbf{G}_{p}\right\}$ are the reciprocal-lattice vectors of the primitive cell, and $\mathbf{k}_{i}^{\alpha}$ is the Bloch $\mathbf{k}$ vector of orbital $i, \operatorname{spin} \alpha$. Then

$$
\left\langle\phi_{j}^{\alpha} \mid \nabla \phi_{i}^{\alpha}\right\rangle=i \delta_{\mathbf{k}_{i}^{\alpha}, \mathbf{k}_{j}^{\alpha}} V_{s} \sum_{\mathbf{G}_{p}}\left(c_{j \mathbf{G}_{p}}^{\alpha}\right)^{*} c_{i \mathbf{G}_{p}}^{\alpha}\left(\mathbf{k}_{i}^{\alpha}+\mathbf{G}_{p}\right) .
$$

This expression together with Eq. (21) is used to evaluate the extra interaction.

\section{Hartree-Fock equations}

Let $f_{i}^{\alpha}$ be the occupation number of state $\phi_{i}^{\alpha}$. Let

$$
\hat{h}_{1}^{\prime}=-\frac{1}{2 \mu} \nabla^{2}-\sum_{\mathbf{R}_{p} \in V_{s}} q_{p} v_{E}\left(\mathbf{x}-\mathbf{R}_{p}\right) .
$$

The expectation value of the Hamiltonian is

$$
\begin{aligned}
\left\langle\Phi\left|\hat{H}^{\prime}\right| \Phi\right\rangle= & \frac{\left(N+N_{p}^{4 / 3} q_{p}^{2}\right) v_{M}}{2}+\sum_{i \alpha}\left\langle\phi_{i}^{\alpha}\left|\hat{h}_{1}^{\prime}\right| \phi_{i}^{\alpha}\right\rangle f_{i}^{\alpha}+\frac{1}{2} \sum_{i \alpha} \sum_{j \beta}\left(\left\langle\phi_{i}^{\alpha} \phi_{j}^{\beta}\left|\hat{v}_{E}\right| \phi_{i}^{\alpha} \phi_{j}^{\beta}\right\rangle-\delta_{\alpha \beta}\left\langle\phi_{i}^{\alpha} \phi_{j}^{\beta}\left|\hat{v}_{E}\right| \phi_{j}^{\beta} \phi_{i}^{\alpha}\right\rangle\right. \\
& \left.-\frac{1}{m_{p}}\left[\left\langle\phi_{i}^{\alpha} \mid \nabla \phi_{i}^{\alpha}\right\rangle \cdot\left\langle\phi_{j}^{\beta} \mid \nabla \phi_{j}^{\beta}\right\rangle-\delta_{\alpha \beta}\left\langle\phi_{i}^{\alpha} \mid \nabla \phi_{j}^{\beta}\right\rangle \cdot\left\langle\phi_{j}^{\beta} \mid \nabla \phi_{i}^{\alpha}\right\rangle\right]\right) f_{i}^{\alpha} f_{j}^{\beta} .
\end{aligned}
$$

To derive the Hartree-Fock (HF) equations, we minimize the expectation value of the Hamiltonian with respect to $\phi_{k}^{\gamma^{*}}$, subject to the constraint that the orbitals remain orthonormal. Introducing Lagrange multipliers $\left\{\lambda_{i j}^{\gamma}\right\}$, we perform an unconstrained minimization of

$$
\mathcal{L}=\left\langle\Phi\left|\hat{H}^{\prime}\right| \Phi\right\rangle-\sum_{\alpha} \sum_{i, j} \lambda_{i j}^{\alpha}\left\langle\phi_{i}^{\alpha} \mid \phi_{j}^{\alpha}\right\rangle f_{i}^{\alpha} f_{j}^{\alpha}
$$

This gives

$$
\begin{aligned}
\hat{h}_{1}^{\prime} \phi_{k}^{\gamma}(\mathbf{x}) & +\sum_{j \beta}\left(\int\left|\phi_{j}^{\beta}\left(\mathbf{x}^{\prime}\right)\right|^{2} v_{E}\left(\mathbf{x}-\mathbf{x}^{\prime}\right) d \mathbf{x}^{\prime} \phi_{k}^{\gamma}(\mathbf{x})-\frac{1}{m_{p}}\left\langle\phi_{j}^{\beta} \mid \nabla \phi_{j}^{\beta}\right\rangle \cdot \nabla \phi_{k}^{\gamma}(\mathbf{x})\right) f_{j}^{\beta} \\
& -\sum_{j}\left(\int \phi_{j}^{\gamma^{*}}\left(\mathbf{x}^{\prime}\right) v_{E}\left(\mathbf{x}-\mathbf{x}^{\prime}\right) \phi_{k}^{\gamma}\left(\mathbf{x}^{\prime}\right) d \mathbf{x}^{\prime} \phi_{j}^{\gamma}(\mathbf{x})-\frac{1}{m_{p}}\left\langle\phi_{j}^{\gamma} \mid \nabla \phi_{k}^{\gamma}\right\rangle \cdot \nabla \phi_{j}^{\gamma}(\mathbf{x})\right) f_{j}^{\gamma}=\sum_{j} \lambda_{k j}^{\gamma} \phi_{j}^{\gamma}(\mathbf{x}) f_{j}^{\gamma} .
\end{aligned}
$$

Writing the left-hand side of Eq. (27) as $\hat{g}_{1}^{\gamma} \phi_{k}^{\gamma}$ and taking the inner product with $\phi_{i}^{\gamma}$, we find that $\left\langle\phi_{i}^{\gamma}\left|\hat{g}_{1}^{\gamma}\right| \phi_{k}^{\gamma}\right\rangle=\lambda_{k i}^{\gamma} f_{i}^{\gamma}$. This is Hermitian and therefore unitarily diagonalizable. Let $\left\{\mathcal{E}_{k}^{\gamma}\right\}$ be the eigenvalues of the matrix $\left\langle\phi_{i}^{\gamma}\left|\hat{g}_{1}^{\gamma}\right| \phi_{k}^{\gamma}\right\rangle$. Unitary transformations among the set of occupied orbitals do not alter the value of the Slater determinant and hence the expectation value of the Hamiltonian. Let us therefore perform the unitary transformation to the set of orbitals in which $\left\langle\phi_{i}^{\gamma}\left|\hat{g}_{1}^{\gamma}\right| \phi_{k}^{\gamma}\right\rangle$ is diagonal, giving the canonical HF equations

$$
\hat{g}_{1}^{\gamma} \phi_{k}^{\gamma}(\mathbf{x})=\mathcal{E}_{k}^{\gamma} \phi_{k}^{\gamma}(\mathbf{x}) \text {. }
$$

These equations should be solved self-consistently because the operator $\hat{g}_{1}^{\gamma}$ depends on the orbital values.

If the orbitals are real or are part of a complex-conjugate pair then the direct part of the extra interaction is zero. This is assumed to be the case in our calculations.

\section{Kohn-Sham equations}

By the Hohenberg-Kohn theorem ${ }^{2}$ the ground-state energy can be written as

$$
\begin{aligned}
E[n]= & \langle\Phi|\hat{T}| \Phi\rangle+\langle\Phi|\hat{A}| \Phi\rangle+E_{H}[n]+E_{\mathrm{ext}}[n]+E_{\mathrm{xc}}[n] \\
& +N v_{M} / 2
\end{aligned}
$$

where $n(\mathbf{x})$ is the ground-state electronic number density in the transformed system, which we assume can be written as the number density of a noninteracting (auxiliary) system with a ground-state Slater wave function $\Phi$ [with orbitals $\left.\left\{\phi_{i}^{\alpha}(\mathbf{x})\right\}\right]$, 


$$
E_{H}[n]=\frac{1}{2} \iint n(\mathbf{x}) n\left(\mathbf{x}^{\prime}\right) v_{E}\left(\mathbf{x}-\mathbf{x}^{\prime}\right) d \mathbf{x} d \mathbf{x}^{\prime}
$$

is the Hartree energy (electrostatic energy arising from the electronic charge density), $E_{\text {ext }}[n]$ is the ground-state expectation of the external potential energy that gives rise to the charge density $n,\langle\Phi|\hat{T}| \Phi\rangle+\langle\Phi|\hat{A}| \Phi\rangle$ is the expectation value of the kinetic-energy operator $\hat{T}=[-1 /(2 \mu)] \Sigma_{i} \nabla_{i}^{2}$ and the extra interaction operator $\hat{A}$, and $E_{\mathrm{xc}}[n]$ is an unknown exchange-correlation (XC) functional, which will be approximated by the LDA functional. By the Hohenberg-Kohn theorem for the noninteracting system, $\langle\Phi|\hat{T}| \Phi\rangle$ and $\langle\Phi|\hat{A}| \Phi\rangle$ are functionals of the density $n$.

As shown by Hohenberg and Kohn, ${ }^{2}$ the energy functional of Eq. (29) should be minimized with respect to $n$ (i.e., with respect to the Slater wave function that gives rise to $n$ ), to yield the ground-state energy and charge density for the given external potential energy,

$$
E_{\text {ext }}[n]=-q_{p} \int n(\mathbf{x}) \sum_{\mathbf{R}_{p} \in V_{s}} v_{E}\left(\mathbf{x}-\mathbf{R}_{p}\right) d \mathbf{x}+\frac{N_{p}^{4 / 3} q_{p}^{2} v_{M}}{2}
$$

due to the positron.

Inserting our expression for the expectation value of the extra interaction operator [Eq. (21)] into Eq. (29) and noting that $n(\mathbf{x})=\sum_{i \alpha}\left|\phi_{i}^{\alpha}(\mathbf{x})\right|^{2}$, we require that $E[n]$ is stationary with respect to changes in the $\left\{\phi_{i}^{\alpha *}(\mathbf{x})\right\}$, subject to the constraint that the orbitals remain orthonormal. This gives the KohnSham equations,

$$
\begin{aligned}
& {\left[-\frac{1}{2 \mu} \nabla^{2}+\int n\left(\mathbf{x}^{\prime}\right) v_{E}\left(\mathbf{x}-\mathbf{x}^{\prime}\right) d \mathbf{x}^{\prime}-q_{p} \sum_{\mathbf{R}_{p} \in V_{s}} v_{E}\left(\mathbf{x}-\mathbf{R}_{p}\right)\right.} \\
& \left.\quad+\frac{\delta E_{\mathrm{xc}}(\mathbf{x})}{\delta n}\right] \phi_{i}^{\gamma}(\mathbf{x})+\frac{1}{m_{p}} \sum_{j} f_{j}^{\gamma}\left\langle\phi_{j}^{\gamma} \mid \nabla \phi_{i}^{\gamma}\right\rangle \cdot \nabla \phi_{j}^{\gamma}(\mathbf{x}) \\
& =\mathcal{E}_{i}^{\gamma} \boldsymbol{\phi}_{i}^{\gamma}(\mathbf{x}) .
\end{aligned}
$$

The charge density in the transformed coordinate system is proportional to the electron-impurity PCF so that the LDA in the transformed coordinates is not equivalent to the LDA in nontransformed coordinates. Nevertheless (i) by the Hohenberg-Kohn theorem the XC energy is a functional of the charge density in the transformed frame, (ii) the LDA is still a local approximation to the XC energy, and (iii) the LDA is, in principle, exact for the HEG. There is no reason to believe that using the LDA in the transformed frame is a worse approximation than using it in the untransformed frame. Unlike the case in DFT calculations with LDA or GGA functionals, however, the single-particle Hamiltonian of Eq. (32) depends explicitly on the orbital values rather than just the density.

\section{Eigenvalue spectrum in transformed coordinates}

Consider the case where $q_{p}=0$, i.e., we perform calculations for a HEG. In this case $\hat{g}_{1}^{\gamma}$ does not contain any direct interaction terms. So

$$
\begin{aligned}
\hat{g}_{1}^{\gamma} \phi(\mathbf{x})= & -\frac{1}{2 \mu} \nabla^{2} \phi-\sum_{j} f_{j}^{\gamma} \int \phi_{j}^{\gamma *}\left(\mathbf{x}^{\prime}\right) v_{E}(\mathbf{x} \\
& \left.-\mathbf{x}^{\prime}\right) \phi\left(\mathbf{x}^{\prime}\right) d \mathbf{x}^{\prime} \phi_{j}^{\gamma}(\mathbf{x})+\frac{1}{m_{p}} \sum_{j} f_{j}^{\gamma}\left\langle\phi_{j}^{\gamma} \mid \nabla \phi\right\rangle \cdot \nabla \phi_{j}^{\gamma}(\mathbf{x}) \\
\equiv & {\left[\hat{t}_{1}+\hat{x}_{1}^{\gamma}+\hat{a}_{1}^{\gamma}\right] \phi(\mathbf{x}) . }
\end{aligned}
$$

For the HEG, the orbitals are plane waves $\exp \left(i \mathbf{G}_{s} \cdot \mathbf{x}\right) / \sqrt{V_{s}}$, where $V_{s}$ is the simulation-cell volume. It is easy to show that

$$
\begin{aligned}
& \exp \left(-i \mathbf{G}_{s} \cdot \mathbf{x}\right) \hat{t}_{1} \exp \left(i \mathbf{G}_{s} \cdot \mathbf{x}\right)=G_{s}^{2} /(2 \mu) \\
& \exp \left(-i \mathbf{G}_{s} \cdot \mathbf{x}\right) \hat{x}_{1}^{\gamma} \exp \left(i \mathbf{G}_{s} \cdot \mathbf{x}\right) \\
& =-\sum_{\mathbf{G}_{s}^{\prime}} \frac{f_{\mathbf{G}_{s}^{\prime}}^{\gamma}}{V_{s}} \exp \left[i\left(\mathbf{G}_{s}-\mathbf{G}_{s}^{\prime}\right) \cdot\left(\mathbf{x}^{\prime}-\mathbf{x}\right)\right] v_{E}\left(\mathbf{x}-\mathbf{x}^{\prime}\right) d \mathbf{x}^{\prime} \\
& =-\frac{1}{V_{s}} \sum_{\mathbf{G}_{s}^{\prime} \neq \mathbf{G}_{s}} \frac{4 \pi f_{\mathbf{G}_{s}^{\prime}}^{\gamma}}{\left|\mathbf{G}_{s}-\mathbf{G}_{s}^{\prime}\right|^{2}} \\
& \exp \left(-i \mathbf{G}_{s} \cdot \mathbf{x}\right) \hat{a}_{1}^{\gamma} \exp \left(i \mathbf{G}_{s} \cdot \mathbf{x}\right) \\
& =\sum_{\mathbf{G}_{s}^{\prime}} \frac{f_{\mathbf{G}_{s}^{\prime}}^{\gamma}}{m_{p} V_{s}} \int \exp \left[i\left(\mathbf{G}_{s}-\mathbf{G}_{s}^{\prime}\right) \cdot\left(\mathbf{x}^{\prime}-\mathbf{x}\right)\right] i \mathbf{G}_{s} d \mathbf{x}^{\prime} \cdot i \mathbf{G}_{s}^{\prime} \\
& =-G_{s}^{2} f_{\mathbf{G}_{s} / m_{p} .}
\end{aligned}
$$

Hence the HF eigenvalues are

$$
\mathcal{E}_{\mathbf{G}_{s}}^{\gamma}=G_{s}^{2}\left(\frac{1}{2 \mu}-\frac{f_{\mathbf{G}_{s}}^{\gamma}}{m_{p}}\right)-\frac{1}{V_{s}} \sum_{\mathbf{G}_{s}^{\prime} \neq \mathbf{G}_{s}} \frac{4 \pi f_{\mathbf{G}_{s}^{\prime}}^{\gamma}}{\left|\mathbf{G}_{s}-\mathbf{G}_{s}^{\prime}\right|^{2}} .
$$

In the ground state, $f_{\mathbf{G}_{s}}=1$ for occupied states and 0 for unoccupied states. Hence, in the infinite-system limit there is a discontinuity of magnitude $k_{F}^{2} / m_{p}$ in the energy band at $k_{F}$. The extra interaction results in a very wide band gap opening up, even though the system is metallic. The same is true for the Kohn-Sham eigenvalues, as shown in Fig. 1.

When the impurity is present (i.e., $q_{p} \neq 0$ ), the transformed system continues to have a huge band gap. This makes the use of a finite temperature to aid the convergence of the self-consistent field process ineffective because the occupancies quickly become either zero or one as the band gap opens up. It is very difficult to change the band occupancy during the calculation. If multiple $\mathbf{k}$ points are used then it is unlikely that the initial (random) set of plane-wave coefficients will lead to the correct number of bands being occupied at each $\mathbf{k}$ point, and hence the calculation becomes trapped in an excited state. For this reason, calculations with multiple $\mathbf{k}$ points do not generally converge to the same result when rerun. This sort of problem can be avoided by using orbitals at $\Gamma$ only.

Of course the existence of a "band gap" is not a physical property of the transformed system. The single-particle eigenvalue spectrum in the transformed system does not cor- 


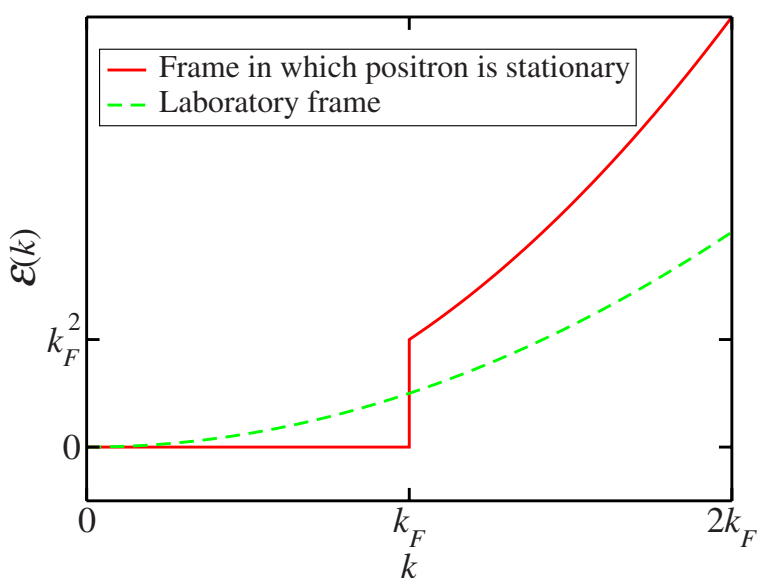

FIG. 1. (Color online) Kohn-Sham eigenvalues for a HEG in the transformed frame in which the "positron" $\left(m_{p}=1\right.$ a.u. $)$ is stationary (solid line) and in the laboratory (untransformed) frame (dashed line).

respond even approximately to differences of eigenvalues of the many-body Hamiltonian $\hat{H}^{\prime}$. For the HEG the eigenvalues of the single-particle kinetic-energy and Coulomb operators are independent of whether the corresponding state is occupied or not; however, the eigenvalue of the extra interaction operator is much lower when the corresponding state is occupied—see Eq. (36)—so that Koopmans' theorem does not apply. Strictly speaking the true energy band is defined by differences between the eigenvalues of $\hat{H}^{\prime}$, which are exactly the same as the eigenvalues of $\hat{H}$, so that the true energy band is not affected by the change in variables.

\section{Symmetry}

The exchange part of the extra interaction only operates between orbitals with the same $\mathbf{k}$. Hence, in a closed-shell system, symmetry-equivalent $\mathbf{k}$ vectors have the same set of eigenvalues. Orbitals at different $\mathbf{k}$ only interact via the charge density. We can therefore use the minimal set of symmetry-inequivalent $\mathbf{k}$ vectors in our DFT calculations.

Symmetry can be broken by having a partially filled shell. In this case one can eliminate $\mathbf{k}$ vectors that are equivalent under the lower symmetry of the open-shell system. Partially filled shells cannot be represented by fractional occupation numbers because the kinetic energy depends linearly on the occupation numbers while the extra interaction depends quadratically on them. A symmetry-reduced set of $\mathbf{k}$ points can only be equivalent to the full set if the occupancies are all either zero or one.

Partially filled shells appear to be undesirable because (i) they generally lead to a violation of the boundary conditions on the wave function as discussed in Sec. III B 2 and (ii) DFT calculations do not converge consistently to the same energy when partially filled shells are used. In our production calculations we have used orbitals at $\Gamma$ only with magic numbers of electrons corresponding to closed-shell configurations for the HEG. So in all of our production calculations the "primitive" cell and "simulation" cells are identical.

\section{Need for the extra interaction terms}

Throughout this work we consider only closed-shell HEG and positron-in-HEG systems. Therefore the extra interaction energy is given by Eq. (21). The extra interaction is dominated by the terms involving high-momentum orbitals, and these should be similar in the HEG and positron-in-HEG systems; hence we may expect a degree of cancellation of the extra interaction energy when the relaxation energy is calculated.

Leung et al. ${ }^{9}$ have argued that the effect of the extra interaction is negligible at low density and have calculated the annihilation rate of a positron in a HEG under this assumption by rescaling the results of calculations performed for a proton (assumed to have an infinite mass) immersed in a HEG. Unfortunately this approximation is a gross violation of the properties of the system as the magnitude of the expectation value of the neglected term is approximately equal to the kinetic energy of the system in the laboratory frame. In order to assess the importance of the extra interaction and to allow comparison with previous studies to be made, we have performed our calculations both with and without the extra interaction.

\section{Density of the electron gas in a finite cell}

Consider an impurity particle immersed in an electron gas of number density $n=3 /\left(4 \pi r_{s}^{3}\right)$. Consider a primitive cell of volume $V_{p}$. Without the impurity, this cell must contain $n V_{p}$ electrons. When the impurity is present, the cell should contain $n V_{p}+q_{p}$ electrons in order to preserve charge neutrality. Provided the cell is sufficiently large, the electron gas will have density $n$ close to the edges of the cell, since the extra electrons will screen the charge of the impurity. The $\left(n V_{p}\right.$ $+q_{p}$ )-electron primitive cell is therefore appropriate for describing the behavior of an impurity immersed in an infinite electron gas of number density $n .^{12}$ In all our calculations, when we refer to an impurity immersed in an $N$-electron HEG of density parameter $r_{s}$, the primitive-cell volume is $V_{p}=(4 / 3) \pi r_{s}^{3}\left(N / N_{p}-q_{p}\right)$.

\section{E. DFT implementation}

\section{Modifications to the castep code}

Henceforth we assume the impurity to be a positron (so $m_{p}=q_{p}=1$ ). The CASTEP (Ref. 13) plane-wave DFT code (version 3.1) was modified to allow the simulation of an electron gas containing a positron. The modified code performs DFT calculations for the system described by the transformed Hamiltonian $\hat{H}^{\prime}$ of Eq. (8). In summary, the following changes to CASTEP have been made: (i) the factor of $1 / \mu$ is included in the kinetic energy; (ii) the exchange part of the extra interaction term is included in the one-particle Hamiltonian and energy expectation value; and (iii) modifications have been made to aid convergence by iterating the orbitals to self-consistency each time the density is updated and to switch on the extra interaction once the orbitals have converged (see Sec. III E 2). 
All our calculations were performed in simple cubic cells. The positron was represented by its bare Coulomb potential in each case.

\section{Strategies for aiding convergence}

When multiple $\mathbf{k}$ points or nonmagic numbers of electrons are used, CASTEP does not consistently converge to the same energy for the reasons discussed in Secs. III C 4 and III C 5. But, even if orbitals at $\Gamma$ only and magic numbers of electrons are used, getting the self-consistent field process to converge can still be problematic if $N$ is large. When the extra interaction terms are omitted from CASTEP, the program has no difficulty converging.

In order to aid convergence in positron-in-HEG calculations, we have made use of two strategies: (A) iterating the orbital coefficients to self-consistency every time the density is updated and (B) converging the orbital coefficients in the absence of the extra interaction, then switching on the extra interaction, and reconverging the orbitals. CASTEP only explicitly symmetrizes the charge density but the extra interaction depends on the orbital coefficients. Hence, when neither of these strategies is used, the one-electron Hamiltonian only has the correct symmetry once self-consistency is achieved. Approach (B) ensures that the initial Hamiltonian in the presence of the extra interaction has the correct symmetry and gives a reasonably good first approximation to the charge density. Approach (A) ensures that the resulting orbitals have the correct symmetry at each iteration. In addition, it was found that using the Kerker ${ }^{14}$ density-mixing scheme increases the likelihood of converging.

\section{CALCULATION OF THE RELAXATION ENERGY}

Let $E\left(N_{0}, M_{0}, V_{p}\right)$ be the ground-state energy per primitive cell of a set of $N_{0}$ particles of unit negative charge and $M_{0}$ particles of unit positive charge per primitive cell. The relaxation energy of a particle of charge 1 a.u. is defined as

$$
\Delta \Omega=E\left(N_{0}+1,1, V_{p}\right)-E\left(N_{0}+1,0, V_{p}\right) .
$$

If the electrons and positron are uncorrelated (but the electrons are correlated with each other) and the system is in its ground state and $\mathbf{k}_{s}=\mathbf{0}$ then the positron energy is zero and the electronic energy is unperturbed by the presence of the positron. In this case the energy per primitive cell of the system with $N_{0}+1$ electrons per primitive cell is simply $E\left(N_{0}+1,0, V_{p}\right)$. On the other hand, when the correlations with the positron are included, the ground-state energy is $E\left(N_{0}+1,1, V_{p}\right)$. So the relaxation energy is the electronpositron correlation energy of the system with $N=N_{0}+1$ electrons per primitive cell.

The low-density limit of the relaxation energy should be the energy of an isolated positronium ion $\left(\mathrm{Ps}^{-}\right)$, a bound state of two electrons and a positron. In practice (i) DFT-LDA is not able to describe the binding of an entire second electron to a positronium atom and the low-density limit of our method will be close to the DFT-LDA energy of a positronium atom; and (ii) this limit appears to be approached very slowly from above, well beyond the range of $r_{s}$ values that we have considered. The calculation of the DFT energy of a positronium atom is discussed in Appendix A.

The first few terms of the exact high-density expansion of the relaxation energy have been calculated ${ }^{15}$ within the random-phase approximation. This expansion is only useful for $r_{s}<0.1$, however.

\section{MD OF ANNIHILATING ELECTRON-POSITRON PAIRS}

\section{A. General expression for the MD}

Suppose a single positron is injected into a sample. Annihilation of the positron with an electron of opposite spin is a second-order process within quantum electrodynamics, leading to the emission of two photons. By contrast, annihilation with an electron of the same spin is a third-order process due to the need to conserve angular momentum, leading to the emission of three photons. The latter process occurs relatively rarely and, in any case, most positron-annihilation experiments are designed to detect the photon pairs produced in opposite-spin annihilation events. ${ }^{6}$ Throughout this work, therefore, we consider only opposite-spin annihilation events.

Consider a HEG with $N_{\uparrow}$ spin-up electrons and $N_{\downarrow}$ spindown electrons in a periodic simulation cell of volume $V_{s}$. Let $N=N_{\uparrow}+N_{\downarrow}$. Let $\left\{\mathbf{r}_{1}, \ldots, \mathbf{r}_{N}\right\}$ be the set of electron coordinates, where the first $N_{\uparrow}$ particles are spin-up electrons. Suppose a single spin-down positron is also present in the electron gas with position vector $\mathbf{s}$. Let $\Psi\left(\mathbf{s} ; \mathbf{r}_{1}, \ldots, \mathbf{r}_{N}\right)$ be the wave function for this system. $\Psi$ is antisymmetric with respect to the exchange of electrons of the same spin. We will make use of electron-positron center-of-mass and difference coordinates, $\overline{\mathbf{r}}_{i} \equiv\left(\mathbf{r}_{i}+\mathbf{s}\right) / 2$ and $\delta \mathbf{r}_{i} \equiv \mathbf{r}_{i}-\mathbf{s}$. In terms of these coordinates we write the wave function as $\Psi\left(\overline{\mathbf{r}}_{1}, \delta \mathbf{r}_{1} ; \mathbf{r}_{2}, \ldots, \mathbf{r}_{N}\right)$. Define the electron-positron center-ofmass momentum wave function to be

$$
\begin{aligned}
& \tilde{\Psi}\left(\overline{\mathbf{p}}_{1}, \delta \mathbf{r}_{1} ; \mathbf{r}_{2}, \ldots, \mathbf{r}_{N}\right) \\
& \quad=\frac{1}{V_{s}} \int \exp \left(-i \overline{\mathbf{p}}_{1} \cdot \overline{\mathbf{r}}_{1}\right) \Psi\left(\overline{\mathbf{r}}_{1}, \delta \mathbf{r}_{1} ; \mathbf{r}_{2}, \ldots, \mathbf{r}_{N}\right) d \overline{\mathbf{r}}_{1}
\end{aligned}
$$

so that

$$
\Psi\left(\overline{\mathbf{r}}_{1}, \delta \mathbf{r}_{1} ; \mathbf{r}_{2}, \ldots, \mathbf{r}_{N}\right)=\sum_{\overline{\mathbf{p}}_{1}} \exp \left(i \overline{\mathbf{p}}_{1} \cdot \overline{\mathbf{r}}_{1}\right) \widetilde{\Psi}\left(\overline{\mathbf{p}}_{1}, \delta \mathbf{r}_{1} ; \mathbf{r}_{2}, \ldots, \mathbf{r}_{N}\right)
$$

(Throughout this paper, integration is assumed to run over a single periodic simulation cell, unless otherwise stated.) Parseval's theorem gives

$$
\int\left|\Psi\left(\overline{\mathbf{r}}_{1}, \delta \mathbf{r}_{1} ; \mathbf{r}_{2}, \ldots, \mathbf{r}_{N}\right)\right|^{2} d \overline{\mathbf{r}}_{1}=V_{s} \sum_{\overline{\mathbf{p}}_{1}}\left|\Psi\left(\overline{\mathbf{p}}_{1}, \delta \mathbf{r}_{1} ; \mathbf{r}_{2}, \ldots, \mathbf{r}_{N}\right)\right|^{2} .
$$

We assume that a positron can only annihilate with an opposite-spin electron when the two particles coincide. We also assume that the probability of annihilation occurring 
when the particles coincide is independent of the positions of the other electrons. Hence the distribution of center-of-mass momenta for annihilating electron-positron pairs is the same as the distribution of the center-of-mass momenta when the positron coincides with an electron of opposite spin.

The unnormalized distribution of the center-of-mass momentum for the positron annihilating with electron 1 is

$$
\int \ldots \int\left|\widetilde{\Psi}\left(\overline{\mathbf{p}}_{1}, \mathbf{0} ; \mathbf{r}_{2}, \ldots, \mathbf{r}_{N}\right)\right|^{2} d \mathbf{r}_{2} \cdots d \mathbf{r}_{N}
$$

By the antisymmetry of the wave function, this is equal to the unnormalized distribution of center-of-mass momenta for annihilating spin-down-positron-spin-up-electron pairs. If we normalize this distribution to $N_{\uparrow}$, we obtain

$$
\rho_{\uparrow}(\overline{\mathbf{p}})=\frac{N_{\uparrow} \int \cdots \int\left|\widetilde{\Psi}\left(\overline{\mathbf{p}}, \mathbf{0} ; \mathbf{r}_{2}, \ldots, \mathbf{r}_{N}\right)\right|^{2} d \mathbf{r}_{2} \cdots d \mathbf{r}_{N}}{\sum_{\overline{\mathbf{p}}} \int \cdots \int\left|\tilde{\Psi}\left(\overline{\mathbf{p}}, \mathbf{0} ; \mathbf{r}_{2}, \ldots, \mathbf{r}_{N}\right)\right|^{2} d \mathbf{r}_{2} \cdots d \mathbf{r}_{N}}=\frac{N_{\uparrow} \int \cdots \int\left|\int \exp \left(-i \overline{\mathbf{p}} \cdot \mathbf{r}_{1}\right) \Psi\left(\mathbf{r}_{1} ; \mathbf{r}_{1}, \ldots, \mathbf{r}_{N}\right) d \mathbf{r}_{1}\right|^{2} d \mathbf{r}_{2} \cdots d \mathbf{r}_{N}}{V_{s} \int \cdots \int\left|\Psi\left(\mathbf{r}_{1} ; \mathbf{r}_{1}, \ldots, \mathbf{r}_{N}\right)\right|^{2} d \mathbf{r}_{1} \cdots d \mathbf{r}_{N}}
$$

A similar expression may be derived for the center-of-mass MD of a spin-up positron annihilating with a spin-down electron, $\rho_{\downarrow}(\overline{\mathbf{p}})$. Unless the system is spin-polarized, however, $\rho(\overline{\mathbf{p}})=\rho_{\uparrow}(\overline{\mathbf{p}})$.

\section{B. Derivation of the MD in a one-electron theory}

Suppose HF theory or DFT has been used to generate a set of orbitals for a positron immersed in a HEG in the transformed coordinate system. In the following we assume $\mathbf{P}=\mathbf{0}$; it is easy to show that if $\mathbf{P} \neq \mathbf{0}$, then the MD is rigidly shifted by $\mathbf{P} /(N+1)$, which is simply a finite-size effect. The many-electron wave function in the untransformed coordinate system can be written as

$$
\Psi\left(\mathbf{s} ; \mathbf{r}_{1}, \ldots, \mathbf{r}_{N}\right)=\frac{1}{\sqrt{N_{\uparrow} ! N_{\downarrow} !}}\left|\begin{array}{ccc}
\phi_{1}^{\uparrow}\left(\mathbf{r}_{1}-\mathbf{s}\right) & \cdots & \phi_{N_{\uparrow}}^{\uparrow}\left(\mathbf{r}_{1}-\mathbf{s}\right) \\
\vdots & & \vdots \\
\phi_{1}^{\uparrow}\left(\mathbf{r}_{N_{\uparrow}}-\mathbf{s}\right) & \cdots & \phi_{N_{\uparrow}^{\uparrow}}^{\uparrow}\left(\mathbf{r}_{N_{\uparrow}}-\mathbf{s}\right)
\end{array}\right|\left|\begin{array}{ccc}
\phi_{1}^{\downarrow}\left(\mathbf{r}_{N_{\uparrow}+1}-\mathbf{s}\right) & \cdots & \phi_{N_{\downarrow}}^{\downarrow}\left(\mathbf{r}_{N_{\uparrow}+1}-\mathbf{s}\right) \\
\vdots & & \vdots \\
\phi_{1}^{\downarrow}\left(\mathbf{r}_{N}-\mathbf{s}\right) & \cdots & \phi_{N_{\downarrow}}^{\downarrow}\left(\mathbf{r}_{N}-\mathbf{s}\right)
\end{array}\right| .
$$

Then the numerator of Eq. (43) is

$$
\begin{aligned}
& \rho_{\uparrow u}(\overline{\mathbf{p}})=\int \cdots \int\left|\int \exp \left(-i \overline{\mathbf{p}} \cdot \mathbf{r}_{1}\right) \Psi\left(\mathbf{r}_{1} ; \mathbf{r}_{1}, \ldots, \mathbf{r}_{N}\right) d \mathbf{r}_{1}\right|^{2} d \mathbf{r}_{2} \cdots d \mathbf{r}_{N}=\frac{1}{N_{\uparrow} ! N_{\downarrow} !} \iint \exp \left[i \overline{\mathbf{p}} \cdot\left(\mathbf{r}_{1}^{\prime}-\mathbf{r}_{1}\right)\right]
\end{aligned}
$$

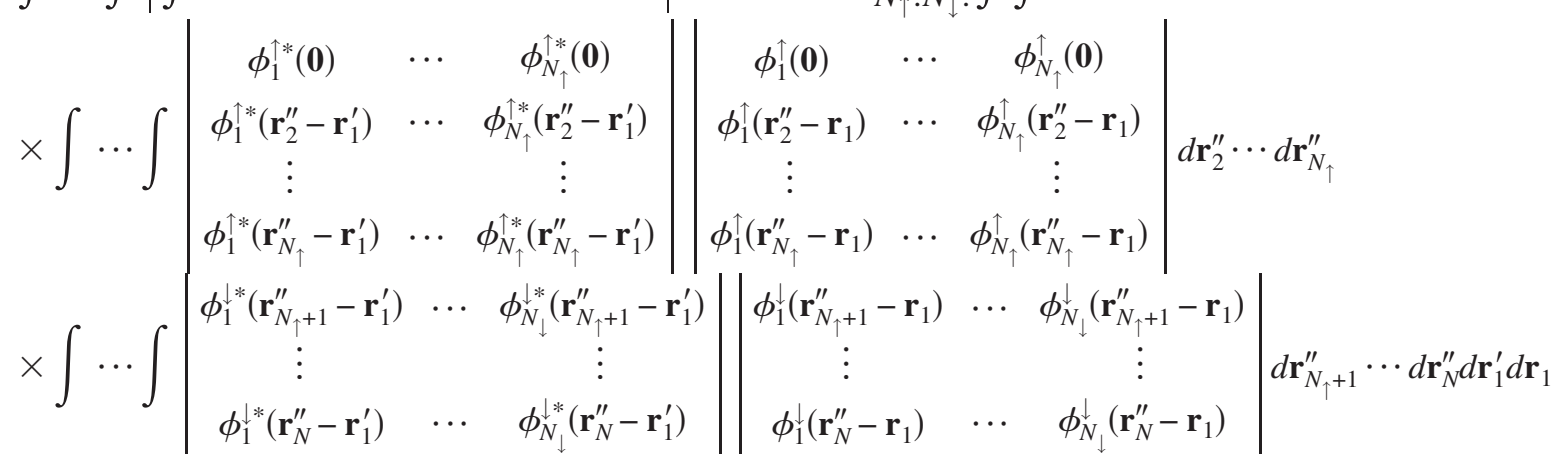

$$
\begin{aligned}
& =\frac{V_{s}}{N_{\uparrow} !} \int \exp (-i \overline{\mathbf{p}} \cdot \mathbf{R}) \int \cdots \int \sum_{i=1}^{N_{\uparrow}} \sum_{j=1}^{N_{\uparrow}}(-1)^{i+j} \boldsymbol{\phi}_{i}^{\uparrow *}(\mathbf{0}) \boldsymbol{\phi}_{j}^{\uparrow}(\mathbf{0}) M_{1 i} N_{1 j} d \mathbf{r}_{2} \cdots d \mathbf{r}_{N_{\uparrow}} \\
& \times\left|\begin{array}{ccc}
\int \phi_{1}^{\downarrow *}(\mathbf{r}) \phi_{1}^{\downarrow}(\mathbf{r}-\mathbf{R}) d \mathbf{r} & \cdots & \int \phi_{N_{\downarrow}}^{\downarrow *}(\mathbf{r}) \phi_{1}^{\downarrow}(\mathbf{r}-\mathbf{R}) d \mathbf{r} \\
\vdots & \vdots \\
\int \phi_{1}^{\downarrow *}(\mathbf{r}) \phi_{N_{\downarrow}}^{\downarrow}(\mathbf{r}-\mathbf{R}) d \mathbf{r} & \cdots & \int \phi_{N_{\downarrow}}^{\downarrow *}(\mathbf{r}) \phi_{N_{\downarrow}}^{\downarrow}(\mathbf{r}-\mathbf{R}) d \mathbf{r}
\end{array}\right|
\end{aligned}
$$

where, in the last step, we have: (i) substituted $\mathbf{R}=\mathbf{r}_{1}-\mathbf{r}_{1}^{\prime}$ and $\mathbf{r}_{i}=\mathbf{r}_{i}^{\prime \prime}-\mathbf{r}_{1}^{\prime} \forall i \in\{2, \ldots, N\}$, allowing us to perform the integral over $\mathbf{r}_{1}^{\prime}$; (ii) made use of the overlap integral theorem for determinants, ${ }^{16}$ (iii) defined $M_{i j}$ to be the $(i, j)$ th minor of 


$$
\left|\begin{array}{ccc}
\phi_{1}^{\uparrow *}(\mathbf{0}) & \cdots & \phi_{N_{\uparrow}}^{\uparrow *}(\mathbf{0}) \\
\phi_{1}^{\uparrow *}\left(\mathbf{r}_{2}\right) & \cdots & \phi_{N_{\uparrow}^{\uparrow *}}\left(\mathbf{r}_{2}\right) \\
\vdots & & \vdots \\
\phi_{1}^{\uparrow *}\left(\mathbf{r}_{N_{\uparrow}}\right) & \cdots & \phi_{N_{\uparrow} *}^{\uparrow *}\left(\mathbf{r}_{N_{\uparrow}}\right)
\end{array}\right| ;
$$

and (iv) defined $N_{i j}$ to be the $(i, j)$ th minor of

$$
\left|\begin{array}{ccc}
\phi_{1}^{\uparrow}(\mathbf{0}) & \cdots & \phi_{N_{\uparrow}}^{\uparrow}(\mathbf{0}) \\
\phi_{1}^{\uparrow}\left(\mathbf{r}_{2}-\mathbf{R}\right) & \cdots & \phi_{N_{\uparrow}}^{\uparrow}\left(\mathbf{r}_{2}-\mathbf{R}\right) \\
\vdots & & \vdots \\
\phi_{1}^{\uparrow}\left(\mathbf{r}_{N_{\uparrow}}-\mathbf{R}\right) & \cdots & \phi_{N_{\uparrow}}^{\uparrow}\left(\mathbf{r}_{N_{\uparrow}}-\mathbf{R}\right)
\end{array}\right| .
$$

For each $i, j \in\left\{1, \ldots, N_{\uparrow}\right\}$, we can use the overlap integral theorem for determinants to determine a $\left(N_{\uparrow}-1\right) \times\left(N_{\uparrow}-1\right)$ matrix $B^{\mathbf{R}}(i, j)$ such that

$$
\operatorname{det}\left[B^{\mathbf{R}}(i, j)\right]=\frac{1}{\left(N_{\uparrow}-1\right) !} \int \cdots \int M_{1 i} N_{1 j} d \mathbf{r}_{2} \cdots d \mathbf{r}_{N_{\uparrow}} .
$$

So, finally, the unnormalized MD of annihilating electronpositron pairs is

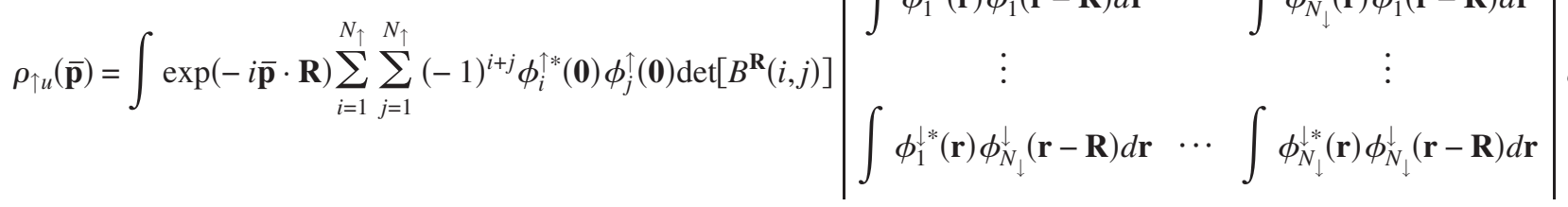

$d \mathbf{R}$.

\section{Implementation of the proposed method using plane-wave orbitals}

\section{Evaluation of the integral over $\mathbf{R}$}

The unnormalized annihilating-pair MD $\rho_{\uparrow u}(\overline{\mathbf{p}})$ is the Fourier transform of

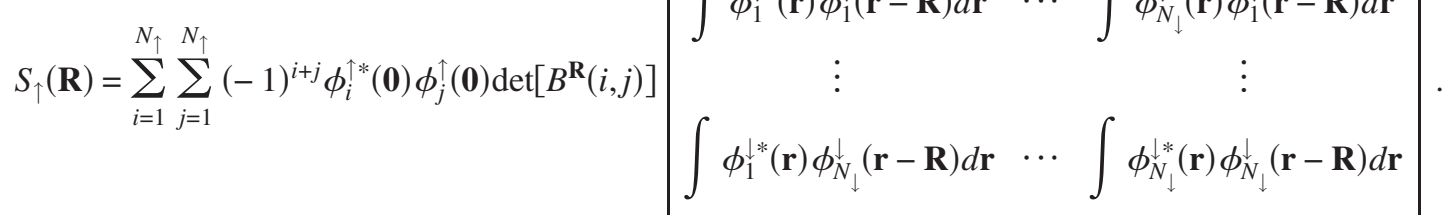

We evaluate $\rho_{\uparrow u}(\overline{\mathbf{p}})$ by a fast Fourier transformation (FFT) method with $S_{\uparrow}(\mathbf{R})$ being calculated by brute force on a grid of points in real space.

Note that $S_{\uparrow}(-\mathbf{R})=S_{\uparrow}^{*}(\mathbf{R})$ corresponding to the fact that $\rho_{\uparrow u}(\overline{\mathbf{p}})$ is real. Furthermore, the symmetry operations of the Bravais lattice can be used to evaluate $S_{\uparrow}(\mathbf{R})$ for all but a symmetry-irreducible set of grid points. Since all lattices have inversion symmetry, $S_{\uparrow}(-\mathbf{R})=S_{\uparrow}(\mathbf{R})$, and so $S_{\uparrow}$ must be real.

\section{Plane-wave bases}

Suppose that each orbital is expanded in a plane-wave basis, thus,

$$
\phi_{i}^{\alpha}(\mathbf{r})=\sum_{\mathbf{G}_{p}} c_{i \mathbf{G}_{p}}^{\alpha} \exp \left[i\left(\mathbf{G}_{p}+\mathbf{k}_{i}^{\alpha}\right) \cdot \mathbf{r}\right],
$$

where $\mathbf{k}_{i}^{\alpha}$ is the Bloch $\mathbf{k}$ vector of the orbital, the $\left\{c_{i \mathbf{G}_{p}}^{\alpha}\right\}$ are a set of plane-wave expansion coefficients, and the $\mathbf{G}_{p}$ vectors are integer multiples of the reciprocal-lattice vectors of the primitive cell. Then the overlaps in the expression for the MD can be evaluated as

$$
\begin{aligned}
& \int \phi_{i}^{\alpha *}(\mathbf{r}) \phi_{j}^{\alpha}(\mathbf{r}-\mathbf{R}) d \mathbf{r} \\
& =V_{s} \sum_{\mathbf{G}_{p}} c_{i \mathbf{G}_{p}^{\alpha *}}^{\alpha *} c_{j \mathbf{G}_{p}}^{\alpha} \exp \left[-i\left(\mathbf{G}_{p}+\mathbf{k}_{i}^{\alpha}\right) \cdot \mathbf{R}\right] \delta_{\mathbf{k}_{i}^{\alpha}, \mathbf{k}_{j}^{\alpha},},
\end{aligned}
$$

where we have made use of the fact that, if the $\mathbf{k}$ points lie on a Monkhorst-Pack ${ }^{17}$ grid, then $\mathbf{G}_{p}^{\prime}-\mathbf{G}_{p}+\mathbf{k}_{j}^{\alpha}-\mathbf{k}_{i}^{\alpha}$ is a reciprocal-lattice vector $\mathbf{G}_{s}$ of the simulation cell, which can only be $\mathbf{0}$ if $\mathbf{G}_{p}=\mathbf{G}_{p}^{\prime}$ and $\mathbf{k}_{j}^{\alpha}=\mathbf{k}_{i}^{\alpha}$. 


\section{Evaluation of the determinants of overlaps}

The overlap determinants $\operatorname{det}\left[B^{\mathbf{R}}(i, j)\right]$ are evaluated using lower triangular-upper triangular (LU) decomposition with CPU requirements that scale as $O\left(N^{3}\right)$ with system size. Alternatively, since only one row of $B^{\mathbf{R}}(i+1, j)$ differs from $B^{\mathbf{R}}(i, j)$, and only one column of $B^{\mathbf{R}}(i, j+1)$ differs from $B^{\mathbf{R}}(i, j)$, the Sherman-Morrison formula ${ }^{18}$ could be used to evaluate all but the first of the determinants for each $\mathbf{R}$. The CPU time for evaluating a determinant using this method would scale as $O\left(N^{2}\right)$ with system size.

\section{Scaling of the method with system size}

For a given $\mathbf{R}$ the time taken to evaluate each of the required orbital overlap integrals $\left[\int \phi_{i}^{\uparrow *}(\mathbf{r}) \phi_{j}^{\uparrow}(\mathbf{r}-\mathbf{R}) d \mathbf{r}, \forall i, j\right]$ scales as $O(N)$ with system size since each element requires a sum over the basis functions to be performed. There are $O\left(N^{2}\right)$ such overlap integrals to evaluate in total.

For a given $\mathbf{R}$ the time taken to compute the determinants $\operatorname{det}\left[B^{\mathbf{R}}(i, j)\right]$ using LU decomposition scales as $O\left(N^{3}\right)$ and there are $O\left(N^{2}\right)$ such determinants to evaluate. Evaluation of the determinants is therefore the rate-determining step at large system sizes. The scaling could be improved as discussed in Sec. V C 3.

The required number of real-space grid points $\mathbf{R}$ grows as $O(N)$. So, overall, the CPU time required by the method is expected to scale as $O\left(N^{6}\right)$. However, the evaluation of the MD only needs to be performed once at the end of the planewave DFT calculation.

\section{Form of the MD and suitable fitting functions}

The MD of an infinite, noninteracting, paramagnetic HEG of density parameter $r_{s}$ is $\rho^{\mathrm{HEG}}(p)=\Theta\left(k_{F}-p\right)$, where the Fermi wave vector is $k_{F}=(9 \pi / 4)^{1 / 3} / r_{s}$. The inclusion of electron-electron interactions, as well as the introduction of a positron, modifies the form of the MD of annihilating electron-positron pairs, but does not change the Fermi wave vector in the limit of infinite system size.

The procedure described in this paper enables us to calculate $\rho(\overline{\mathbf{p}})$ for those particular values of $\overline{\mathbf{p}}$ that are commensurate with a finite simulation cell. By fitting a smooth function of $\bar{p}$ to our results, we may approximate the continuous MD of the infinite system. For finite systems, we determine whether the discrete momenta lie before or after the Fermi edge by considering whether or not the corresponding orbitals would be occupied in a finite, noninteracting, paramagnetic HEG.

Assuming the MD to be analytic inside the Fermi sphere, only even powers of $\bar{p}$ can appear in the Taylor expansion of the MD about $\bar{p}=0$. Following Kahana, ${ }^{19}$ we perform a leastsquares fit of a quartic function to our discrete MD results before the Fermi edge,

$$
\rho^{\operatorname{model}}(\bar{p})=A+B \bar{p}^{2}+C \bar{p}^{4},
$$

where $A, B$, and $C$ are parameters to be determined by fitting. After the Fermi edge, we assume the MD to fall off as

$$
\rho^{\text {model }}(\bar{p})=a \exp (-s \bar{p}),
$$

where $a$ and $s$ are parameters to be determined by fitting to the discrete MD results after the Fermi edge. (The decay of the DFT MD was found to be exponential rather than algebraic in numerical tests.)

Having fitted the model functions to the pre-edge and postedge results, we plot the fitted functions, but move the Fermi edge to its correct position in the infinite-system limit.

\section{ELECTRON-POSITRON PCF}

The positron-spin-up-electron PCF is defined to be

$$
g^{\uparrow}(\mathbf{r}, \mathbf{s})=\frac{\rho_{2 p}^{\uparrow}(\mathbf{r}, \mathbf{s})}{\rho_{1}^{\uparrow}(\mathbf{r}) \rho_{p}(\mathbf{s})},
$$

where

$$
\rho_{2 p}^{\uparrow}(\mathbf{r}, \mathbf{s})=\frac{N_{\uparrow} N_{p} \int \ldots \int\left|\Psi\left(\mathbf{s} ; \mathbf{r}, \mathbf{r}_{2}, \ldots, \mathbf{r}_{N}\right)\right|^{2} d \mathbf{r}_{2} \cdots d \mathbf{r}_{N}}{\int \cdots \iint\left|\Psi\left(\mathbf{s} ; \mathbf{r}_{1}, \ldots, \mathbf{r}_{N}\right)\right|^{2} d \mathbf{r}_{1} \cdots d \mathbf{r}_{N} d \mathbf{s}}
$$

is the electron-positron pair density, where $N_{p}=V_{s} / V_{p}$ is the number of primitive cells in the simulation cell, while $\rho_{1}^{\uparrow}(\mathbf{r})=N_{\uparrow} / V_{s}$ is the spin-up electron density, and $\rho_{p}(\mathbf{s})=1 / V_{p}$ is the positron density. The system is homogeneous, so $g^{\uparrow}(\mathbf{r}, \mathbf{s})=g^{\uparrow}(\mathbf{r}-\mathbf{s})$. Hence the PCF is given by

$$
g^{\uparrow}(\mathbf{r}-\mathbf{s})=\frac{V_{s}^{2} \int \ldots \int\left|\Psi\left(\mathbf{s} ; \mathbf{r}, \mathbf{r}_{2}, \ldots, \mathbf{r}_{N}\right)\right|^{2} d \mathbf{r}_{2} \cdots d \mathbf{r}_{N}}{\int \ldots \iint\left|\Psi\left(\mathbf{s} ; \mathbf{r}_{1}, \ldots, \mathbf{r}_{N}\right)\right|^{2} d \mathbf{r}_{1} \cdots d \mathbf{r}_{N} d \mathbf{s}} .
$$

Suppose the wave function has the form given in Eq. (44) with orthogonal orbitals. Then

$$
g^{\uparrow}(\mathbf{r}-\mathbf{s})=\frac{V_{s}}{N_{\uparrow}} \sum_{i=1}^{N_{\uparrow}} \frac{\left|\phi_{i}^{\uparrow}(\mathbf{r}-\mathbf{s})\right|^{2}}{\left\langle\phi_{i}^{\uparrow} \mid \phi_{i}^{\uparrow}\right\rangle} .
$$

From Eq. (57) and the fact that the positron density is constant, the normalization condition on the PCF in a finite cell $^{20}$ can be seen to be $\int\left[g^{\uparrow}(\mathbf{r})-1\right] d \mathbf{r}=0$. The shape of the primitive cell results in a slight anisotropy in the PCF at large distances from the positron. We have therefore spherically averaged the PCF by integrating $g^{\uparrow}(\mathbf{r}) /\left(4 \pi r^{2}\right)$ over the surface of a sphere of radius $r$. The integration method described in Ref. 21 (rule 4) was used.

For a paramagnetic HEG (which is the only case we have considered in the calculations reported here), the total PCF is simply $g(r)=g^{\uparrow}(r)$.

\section{ELIMINATING SOURCES OF ERROR IN POSITRON-IN-HEG CALCULATIONS}

\section{A. Relaxation energy}

\section{Finite-basis errors}

The finite-basis error in the relaxation energy is found to fall off as $E_{\text {cut }}^{-4 / 3}$ in numerical tests, as shown in Fig. 2. Hence 

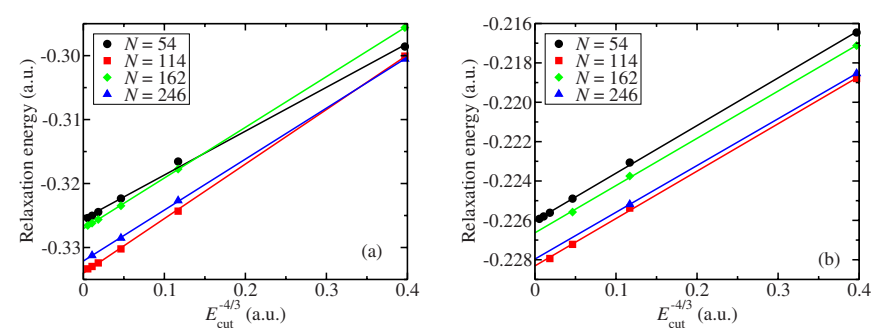

FIG. 2. (Color online) Dependence of the relaxation energy on the plane-wave cutoff energy $E_{\text {cut }}$ for positrons immersed in HEGs of different system size $N$ at density parameter (a) $r_{s}=2$ and (b) $r_{s}=5$.

the relaxation energy can be extrapolated to basis-set completeness. We have done this in all our production calculations.

\section{Finite-size effects}

Our DFT calculations suffer from two sources of finitesize errors: (i) neighboring images of the positron interact with one another and (ii) momentum quantization (shellfilling) effects are present. Shell-filling effects are large in metallic systems such as the HEG, but they tend to cancel out of quantities such as the relaxation energy, because exactly the same simulation cell is used for the calculations with and without the positron.

In practice, for the system sizes we have studied (up to a few hundred electrons) finite-size errors are predominantly due to shell-filling effects for $N>50$ at intermediate and high densities, as demonstrated by the oscillatory behavior of the relaxation energy as a function of $N$, see Fig. 3. Finite-size errors are smaller at lower densities. We have attempted to reduce finite-size errors by discarding data at small $N$ (i.e., $N<114$ for $1 \leq r_{s} \leq 3.5, N<54$ for $4 \leq r_{s} \leq 6.5$, and $N<14$ for $\left.r_{s} \geq 7\right)$ and then averaging the remaining data.

The bias resulting from finite-basis errors is made negligible by extrapolation but finite-size errors affect the second or third significant figure of the relaxation energy at high densities. At low densities, both finite-basis and finite-size errors are small.

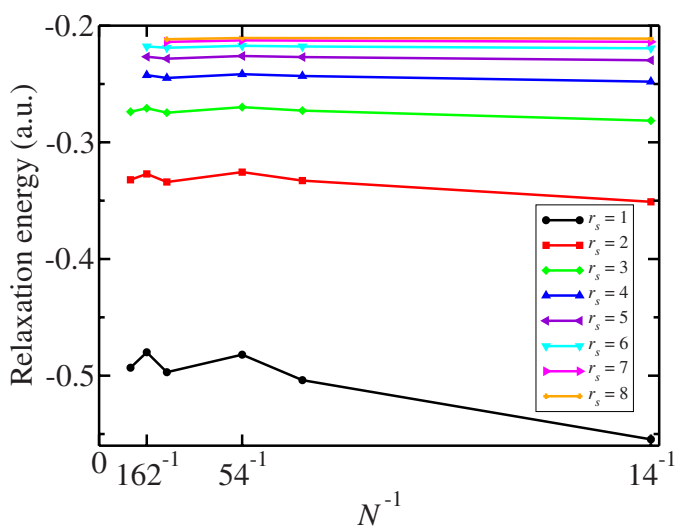

FIG. 3. (Color online) Relaxation energy against inverse system size $N^{-1}$ for positrons immersed in HEGs at different density parameters $r_{s}$. The relaxation energies have been extrapolated to basisset completeness in each case.

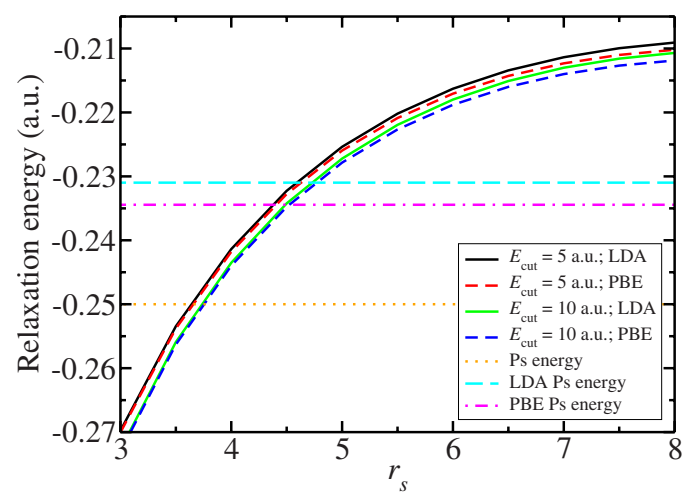

FIG. 4. (Color online) Relaxation energy against $r_{s}$ for a positron immersed in a 114-electron HEG.

\section{XC functional}

We have verified that the relaxation energies calculated using the LDA and Perdew-Burke-Ernzerhof (PBE) ${ }^{22}$ generalized-gradient-approximation $\mathrm{XC}$ functionals are almost identical, see Fig. 4. All other results quoted in this paper were calculated within the LDA.

\section{B. Momentum density}

\section{Finite-basis errors}

Numerical tests suggest that

$$
\rho_{E_{\mathrm{cut}}}(\bar{p})=\rho_{\infty}(\bar{p})+a_{1}(\bar{p}) E_{\mathrm{cut}}^{-1}+a_{3 / 2}(\bar{p}) E_{\mathrm{cut}}^{-3 / 2}
$$

is a suitable fitting function for extrapolating the momentum density at each $\bar{p}$ to basis-set completeness, see Fig. 5.

\section{Finite-size errors}

As the system size is increased the density of momenta $\bar{p}$ at which the MD is evaluated increases. Furthermore, oscil-
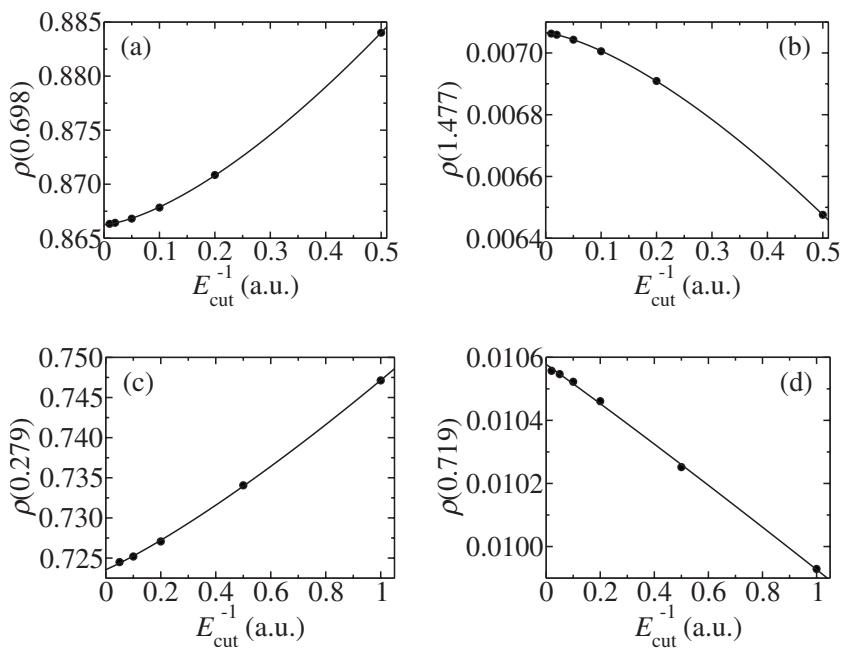

FIG. 5. Dependence of the annihilating-pair MD at particular momenta on the plane-wave cutoff energy $E_{\text {cut }}$ for positrons immersed in (a) a 114-electron HEG of density parameter $r_{s}=2$, (b) a 162-electron HEG at $r_{s}=2$, (c) a 114-electron HEG at $r_{s}=5$, and (d) a 54-electron HEG at $r_{s}=5$. The solid line shows the fit to Eq. (59) in each case. 

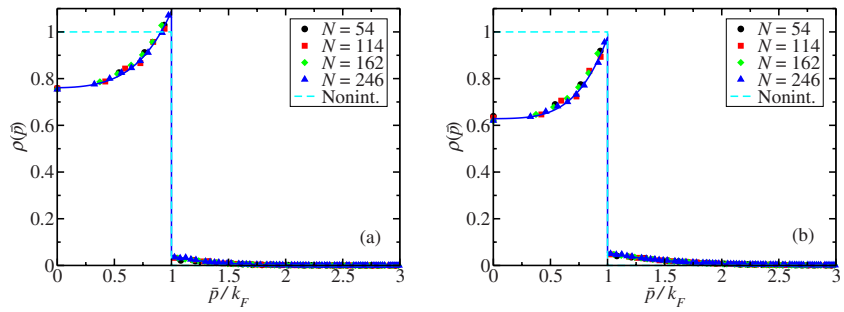

FIG. 6. (Color online) Annihilating-pair MDs for positrons immersed in HEGs of density parameter (a) $r_{s}=2$ and (b) $r_{s}=5$. The MDs have been extrapolated to basis-set completeness. The curves labeled "Nonint." show the MD of a free-electron gas.

lations in the MD due to shell-filling effects decrease. For this reason we simply fit $\rho^{\operatorname{model}}(\bar{p})$ to the MD at the largest system size for which data are available at each density to obtain our final results. Results obtained at a range of system sizes are shown in Fig. 6. It is clear that the momentum density is well converged with respect to the system size.

\section{FFT grid size}

It was verified that our MD data are converged with respect to the FFT grid on which $S_{\uparrow}(\mathbf{R})$ is calculated. A $16 \times 16 \times 16$ grid was used in all of our calculations.

\section{Electron-positron PCF and contact PCF}

\section{Finite-basis errors}

The PCF should satisfy the Kimball cusp condition ${ }^{23}$ at the positron but it cannot do so when the orbitals are expanded in a finite number of plane waves. This leads to significant finite-basis errors in the PCF, as can be seen in Fig. 7. We have therefore used a scheme for imposing the Kimball condition on the PCF, which is described in Appendix B. The remaining finite-basis errors in the cusp-corrected PCF are relatively small, as can be seen in Fig. 7 .

Numerical tests show that the error in the noncuspcorrected contact PCF $g(0)$ falls off as $E_{\text {cut }}^{-1 / 2}$, see Fig. 8. It is therefore possible to extrapolate the contact PCF to basis-set completeness without adding cusp-correction functions. The fit can be considerably improved by including a term going as $E_{\text {cut }}^{-1}$, i.e., we fit $g_{E_{\text {cut }}}(0)$ to

$$
g_{E_{\text {cut }}}(0)=g_{\infty}(0)+a_{1 / 2} E_{\text {cut }}^{-1 / 2}+a_{1} E_{\text {cut }}^{-1} \text {. }
$$

Although the extrapolation of the noncusp-corrected PCF to basis-set completeness using Eq. (60) works well at $r=0$, it
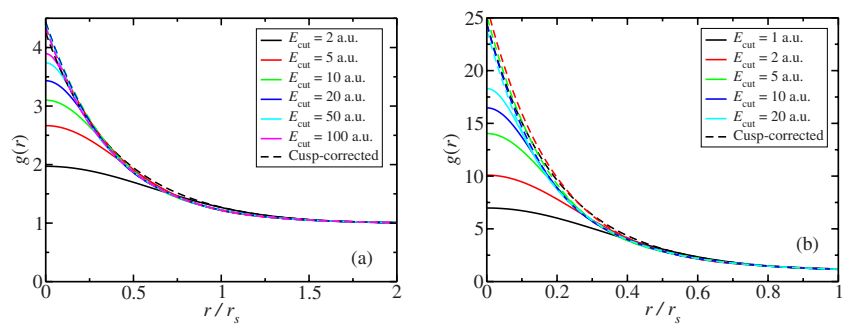

FIG. 7. (Color online) PCFs for positrons immersed in 114electron HEGs of density parameter (a) $r_{s}=2$ and (b) $r_{s}=5$. The solid lines show the uncorrected PCFs and the dashed lines show the cusp-corrected PCFs.
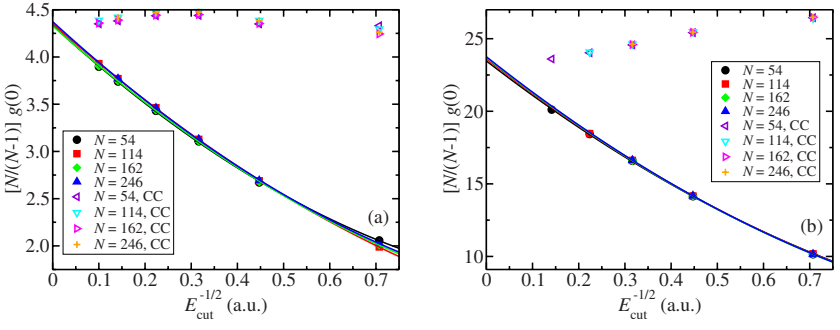

FIG. 8. (Color online) Dependence of the contact PCF $g(0)$ on the plane-wave cutoff energy $E_{\text {cut }}$ for positrons immersed in HEGs of density parameter (a) $r_{s}=2$ and (b) $r_{s}=5$. The data have been multiplied by $N /(N-1)$, as discussed in Sec. VII C 2 , and the data labeled "CC" have been cusp-corrected using the scheme described in Appendix B.

does not work so well at other $r$, where the dependence of $g(r)$ on $E_{\text {cut }}$ is more complex. If one uses the extrapolation scheme of Eq. (60) anyway, the resulting PCF does not satisfy the Kimball condition.

It can be seen in Fig. 8 that the error in the cusp-corrected contact PCF falls off relatively rapidly. However, in our final results for the contact PCF we have used the noncuspcorrected data extrapolated to basis-set completeness because these data have been subject to less processing and hence there is less noise in the estimated contact PCF.

\section{Finite-size errors}

In an infinite electron gas, $g^{\uparrow}(|\mathbf{r}-\mathbf{s}|) \rightarrow 1$ as $|\mathbf{r}-\mathbf{s}| \rightarrow \infty$. For a finite electron gas, however, the density of spin-up electrons far from the positron is effectively reduced because on average $N_{\uparrow} / N$ spin-up electrons bind to each positron. So $\rho_{1 p}^{\uparrow}(\mathbf{r}, \mathbf{s}) \rightarrow\left[\left(N_{\uparrow} / N_{p}-N_{\uparrow} / N\right) / V_{p}\right] \times\left[1 / V_{p}\right]$. Hence $g^{\uparrow}(|\mathbf{r}-\mathbf{s}|)$ $\rightarrow\left(N-N_{p}\right) / N$. This suggests that to reduce the finite-size errors in the PCF, the PCF obtained in a finite, $N$-electron cell in a nonspin-polarized calculation should be multiplied by $N /\left(N-N_{p}\right)$, despite the fact that this will violate the normalization condition ${ }^{20}$ on the finite-system PCF.

Once this finite-size correction has been applied, the PCF is extremely well converged as a function of system size, as can be seen in Fig. 9. For the contact PCF we simply average over the noncusp-corrected contact PCF values extrapolated to basis-set completeness obtained with $N>100$. For the whole PCF, we note that it is more important to use a large
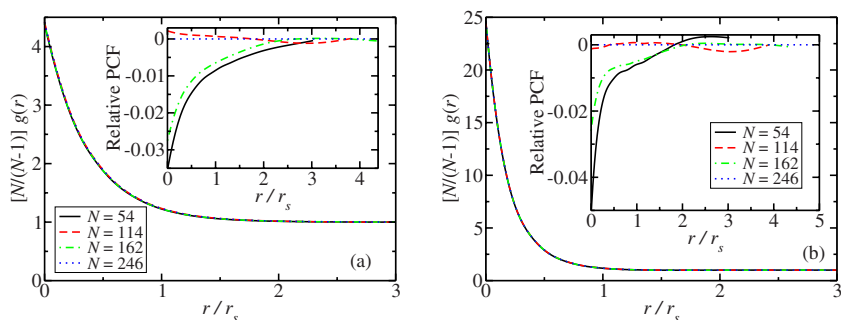

FIG. 9. (Color online) Cusp-corrected PCFs for positrons immersed in HEGs of density parameter (a) $r_{s}=2$ and (b) $r_{s}=5$. The data have been multiplied by $N /(N-1)$. The plane-wave cutoff energy was 50 and 10 a.u. at $r_{s}=2$ and $r_{s}=5$, respectively. The insets show the PCFs relative to that obtained with $N=246$ electrons. 


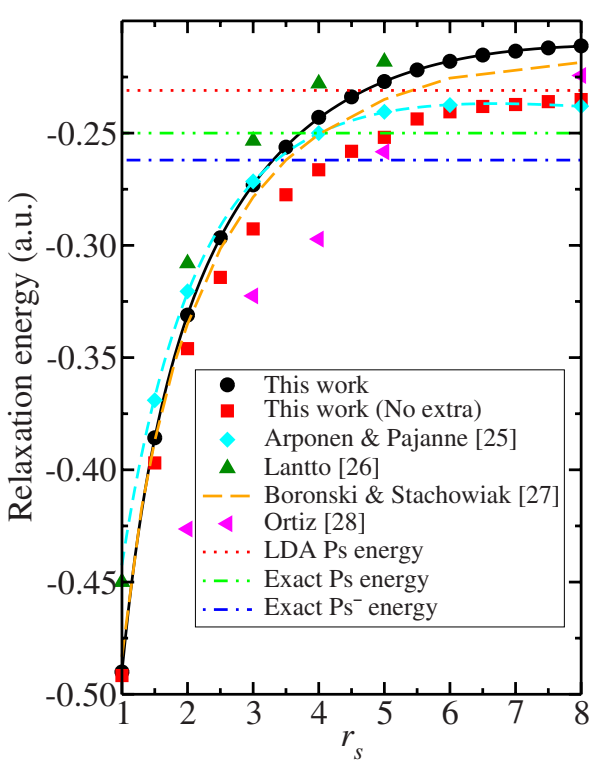

FIG. 10. (Color online) Relaxation energy against density parameter. Results obtained with $N=114,162$, and 246 electrons for $1 \leq r_{s} \leq 3.5$, with $N=54,114$, and 162 electrons for $4 \leq r_{s} \leq 6.5$, and with $N=14,54$, and 114 electrons for $7 \leq r_{s} \leq 8$ were averaged to obtain the final results. Also shown are the relaxation energies obtained by other authors (Refs. 25-28). The dashed line following the data of Arponen and Pajanne (Ref. 25) is the widely used fit of Boroński and Nieminen (Ref. 7). The energy of the $\mathrm{Ps}^{-}$ion is taken from Ref. 24. The curve labeled "No extra" was obtained without the extra interaction term in the transformed Hamiltonian.

plane-wave cutoff energy than a large number of electrons $N$, and hence we report cusp-corrected PCFs obtained with $N$ $=54$ electrons at the largest plane-wave cutoff energy available at each density.

\section{RESULTS}

\section{A. Relaxation energy}

The fit to our relaxation-energy data shown in Fig. 10 is

$$
\Delta \Omega\left(r_{s}\right)=\frac{A_{-1} r_{s}^{-1}+A_{0}+A_{1} r_{s}-0.262005 B_{2} r_{s}^{2}}{1+B_{1} r_{s}+B_{2} r_{s}^{2}},
$$

where $A_{-1}=-0.28877, A_{0}=-0.22339, A_{1}=0.011536, B_{1}$ $=0.012331$, and $B_{2}=0.020016$. This fitting form tends to the energy of the positronium ion $\left(\mathrm{Ps}^{-}\right)$at low density ${ }^{24}$ and could be used as the LDA electron-positron correlation functional in a two-component DFT calculation for a positron in a real system. Equation (10) does not yield the exact highdensity behavior calculated within the random-phase approximation, although this is only relevant for $r_{s}<0.1 .^{15}$

Our relaxation-energy results are in reasonable agreement with the many-body-theory results of Refs. 25 and 27 but are in clear disagreement with the quantum Monte Carlo (QMC) data of Ref. 28. The orbitals in the trial wave functions used in the QMC calculations reported in Refs. 28 and 29 were single plane waves for the electrons and the positron, which do not allow for the strong pairing that occurs between the electrons and the positron at low density. In common with

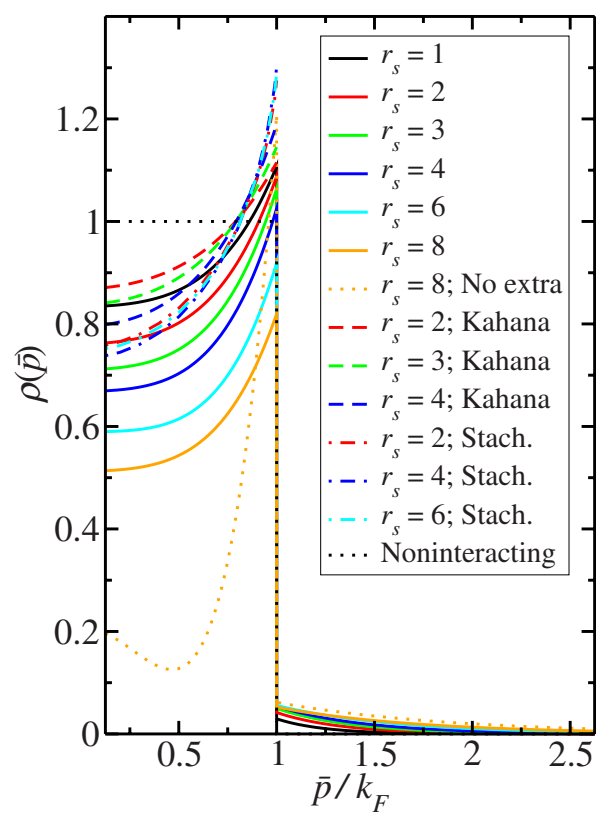

FIG. 11. (Color online) Annihilating-pair MDs $\rho(\bar{p})$ for different densities. The solid lines show the MDs calculated with the extra interaction included; the dotted line shows the MD calculated at $r_{s}=8$ without the extra interaction. Model functions have been fitted to the results obtained with $N=406$ electrons at $r_{s}=1, N=246$ electrons for $2 \leq r_{s} \leq 7$, and $N=162$ electrons at $r_{s}=8$. MD data calculated by Kahana (Ref. 19) and Stachowiak (Ref. 30) using other approaches are also shown. The dotted curve labeled No extra was obtained without the extra interaction term in the transformed Hamiltonian.

the most accurate many-body-theory results, our relaxation energy is higher than the energy of a positronium atom at the lowest density considered of $r_{s}=8$. Neglecting the extra interaction as suggested by Leung et al..$^{9}$ leads to relaxation energies that increase monotonically towards the DFT-LDA energy of a positronium atom.

\section{B. Annihilating-pair MD}

The annihilating-pair MD depends sensitively on the accuracy of the correlated electron-positron pairing orbital $\phi_{i}(\mathbf{x})$ and we expect that our fully self-consistent treatment of the pairing orbitals will be more accurate than previous approaches..$^{19,30}$ As the system size is increased, the momenta at which the MD is defined become more finely spaced and the finite-size errors at each point are reduced. We have therefore fitted a model curve to our MD data obtained at the largest system size available at each density. We have verified that our results are well converged with respect to system size.

Electron-positron annihilating-pair MDs at different densities are plotted in Fig. 11. The normalization is chosen such that $\int_{0}^{\infty} 4 \pi \bar{p}^{2} \rho(\bar{p}) d \bar{p}=(4 / 3) \pi k_{F}^{3}$. Our results clearly show the enhancement of the annihilating-pair MD at the Fermi edge predicted by Kahana ${ }^{19}$ but our MD data differ quantitatively from the previous results ${ }^{19,30}$ for $1 \leq r_{s} \leq 8$. In this range, we find the greatest enhancement of the MD at the Fermi edge at $r_{s}=1$, whereas the previous works ${ }^{19,30}$ found the enhance- 


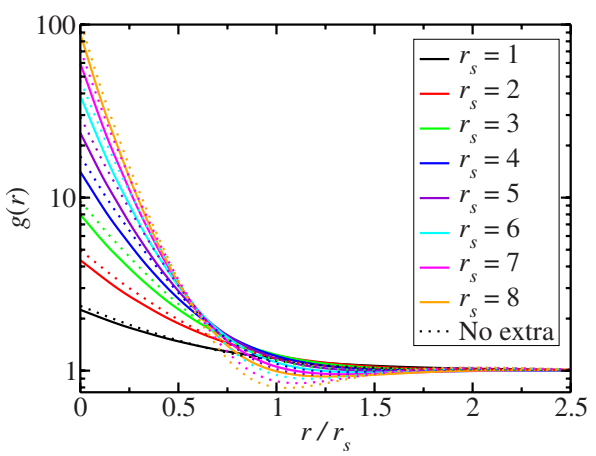

FIG. 12. (Color online) Electron-positron PCF for positrons immersed in HEGs of different density parameter $r_{s}$. The solid line shows results obtained with the extra interaction terms present; the dotted lines show results obtained without the extra interaction. The data are for 54-electron HEGs and the finite-size correction discussed in Sec. VII C 2 has been applied. The cusp correction described in Appendix B has been applied. The plane-wave cutoff energies are 400, 100, 100, 50, 50, 20, 20, and 20 a.u. at $r_{s}=1,2,3$, $4,5,6,7$, and 8 , respectively. The dotted curves labeled No extra were obtained without the extra interaction term in the transformed Hamiltonian.

ment of the MD to increase when the density is lowered. The previous works ${ }^{19,30}$ did not report the weight in the MDs after the Fermi edge. In our calculations the exponential tail of the MD after the Fermi edge carries an increasing amount of weight as the density is lowered, which is responsible for the decrease in the enhancement that we find at the Fermi edge. We find the exponent $s$ in Eq. (54) to be on the order of $r_{s}$ over the range of densities we have studied. It is essential to include the extra interaction when calculating the MD; omitting it results in a dramatic increase in the enhancement at the Fermi edge and a substantial transfer of weight to momenta after the Fermi edge, as shown in Fig. 11. The Kohn-Sham orbitals do not describe the electron-electron correlation. Such correlation effects (i) tend to oppose the enhancement at the Fermi edge, particularly at low densities, and (ii) introduce an algebraically decaying tail in the MD. ${ }^{31}$

\section{PCF}

Electron-positron PCFs at different densities are plotted in Fig. 12. It is clear that the contact PCF increases rapidly as the density is reduced. For densities lower than $r_{s}=3$ there is a region in which $g(r)<1$, so the probability of finding an electron is reduced in this region. At higher densities this region is either nonexistent or very shallow and far from the positron. The extra interaction has relatively little effect at short range but results in a more pronounced dip in the PCF at longer range.

\section{Contact PCF}

The electron-positron contact PCF is plotted in Fig. 13. Adapting the fitting form of Ref. 7 slightly, we represent our contact PCF data by

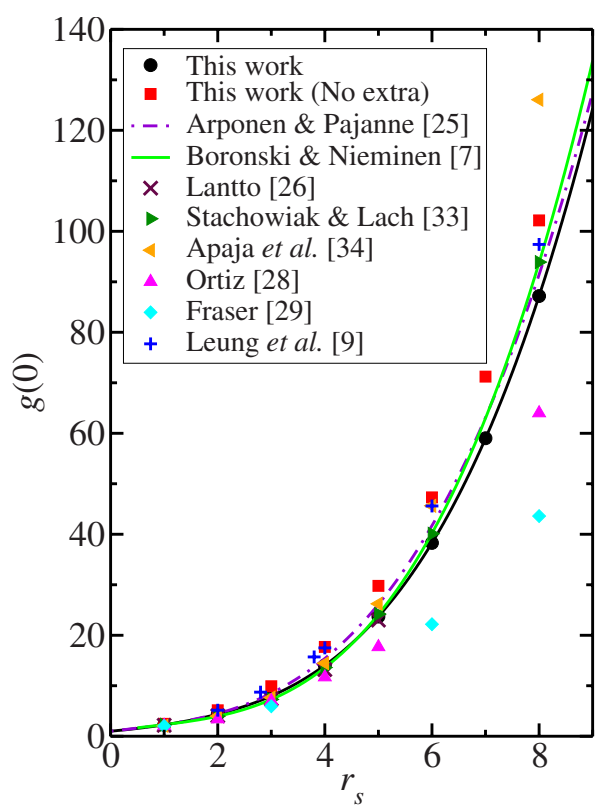

FIG. 13. (Color online) Electron-positron contact PCF $g(0)$ against density parameter $r_{s}$. The results were obtained by averaging the $g(0)$ values obtained in cells with $N=114,162$, and 246 electrons for $1 \leq r_{s} \leq 7$ and $N=114$ and 162 at $r_{s}=8$. Various contact PCF results from the literature are also shown. The results of Leung et al. (Ref. 9) were obtained by rescaling the data for a proton in a HEG obtained by Almbladh et al. (Ref. 32). The curve labeled No extra was obtained without the extra interaction term in the transformed Hamiltonian.

$$
\begin{aligned}
g(0)= & 1+1.23 r_{s}+a_{3 / 2} r_{s}^{3 / 2}+a_{2} r_{s}^{2}+a_{7 / 3} r_{s}^{7 / 3}+a_{8 / 3} r_{s}^{8 / 3} \\
& +0.173694 r_{s}^{3},
\end{aligned}
$$

where $a_{3 / 2}=-1.56672, a_{2}=4.16983, a_{7 / 3}=-3.579$, and $a_{8 / 3}$ $=0.836389$ are determined by fitting. Equation (62) satisfies both the high-density (random-phase approximation) ${ }^{15}$ limiting behavior and the low-density $\left(\mathrm{Ps}^{-}\right)$limiting behavior discussed in Appendix C.

The extra interaction reduces the contact PCF at all the densities we have studied, by about $8 \%$ at $r_{s}=1$, rising to about $15 \%$ at $r_{s}=8$. Our electron-positron PCF results are in reasonably good agreement with the many-body-theory results in the literature, ${ }^{25,26,33,34}$ but are in strong disagreement with the QMC results, ${ }^{28,29}$ which give much smaller values for the reasons discussed earlier.

The results obtained by Leung et al. ${ }^{9}$ are, in principle, equivalent to our results without the extra interaction. The agreement is excellent at high density but less good at low density. The difference could be due to the use of different parametrizations of the LDA exchange-correlation functional, or finite-size errors, or errors arising from the process of extrapolation to basis-set completeness.

\section{CONCLUSIONS}

In summary, we have used a modified one-component DFT code to calculate the relaxation energy, contact PCF, and annihilating-pair MD of a single positron in a HEG by 
working in the frame in which the positron is stationary. A mean-field theory in the transformed frame gives orbitals $\phi\left(\mathbf{r}_{i}-\mathbf{s}\right)$, where $\mathbf{r}_{i}$ and $\mathbf{s}$ are electron and positron positions, and therefore electron-positron correlations are included explicitly. It is interesting to observe that the data required to parametrize a two-component exchange-correlation density functional can be obtained using one-component DFT calculations. Our results for the positron relaxation energies and annihilation rates are in broad agreement with earlier theoretical work based on many-body-theory results, except the QMC results ${ }^{28,29}$ which are based on inadequate trial wave functions. The annihilating-pair MDs are particularly sensitive to the description of the electron-positron correlation. We have reported MDs for a wider range of densities than previous studies, and our calculations extend to momenta after the Fermi edge, where we find an exponential decay of the MD with momentum. The MD bears the imprint of the DFT description of the electron-electron correlations, and including an explicit many-body description would give a power-law decay of the MD after the Fermi edge.

\section{ACKNOWLEDGMENTS}

We acknowledge financial support from the Leverhulme Trust, Jesus College, Cambridge, and the U.K. Engineering and Physical Sciences Research Council (EPSRC). We thank P.J. Hasnip for assistance with the CASTEP code.

\section{APPENDIX A: EVALUATING THE DFT ENERGY OF AN ISOLATED POSITRONIUM ATOM}

We have used the bare Coulomb potential and a reduced mass of $1 / 2$ to calculate the DFT energy of an isolated positronium atom. Fixed-occupancy calculations were performed in cubic cells at the $\Gamma$ point. For each cell length $L_{\text {cell }}$, the energy was extrapolated to basis-set completeness by fitting to

$$
E\left(E_{\text {cut }}, L_{\text {cell }}\right)=E\left(\infty, L_{\text {cell }}\right)+a_{L_{\text {cell }}} E_{\text {cut }}^{\gamma_{L_{\text {cell }}}},
$$

where $a_{L_{\text {cell }}}$ is a fitting parameter and the exponent $\gamma_{L_{\text {cell }}}$ turned out to be about 1.44 at all cell lengths.

The resulting energies were then extrapolated to infinite cell size by fitting to

$$
E\left(\infty, L_{\text {cell }}\right)=E(\infty, \infty)+b \exp \left(s L_{\text {cell }}\right),
$$

where $b$ and $s$ are fitting parameters. The exponential form is appropriate since the positronium wave function falls off exponentially.

The DFT-LDA energy of an isolated positronium atom was found to be -0.23098 a.u. while the DFT-PBE energy was found to be -0.23444 a.u. These are a little smaller in magnitude than the exact value of -0.25 a.u.

\section{APPENDIX B: KIMBALL CUSP CONDITIONS ON THE ELECTRON-POSITRON PCF}

The Kimball cusp condition ${ }^{23}$ on the electron-positron PCF $g^{\uparrow}(r)$ is

$$
\left(\frac{\partial \bar{g}^{\uparrow}}{\partial r}\right)_{r=0}=-g^{\uparrow}(0),
$$

where the bar denotes a spherical average. [To prove this, note that $|\bar{\phi}(r)|^{2}=\overline{|\phi(\mathbf{r})|^{2}}+O\left(r^{2}\right)$, then differentiate the spherical average of Eq. (57) with respect to $r$ and make use of the Kato cusp conditions ${ }^{35}$ on the wave function.]

The PCF evaluated with orbitals expanded in plane waves clearly cannot satisfy Eq. (B1) for finite plane-wave cutoff energies $E_{\text {cut }}$. This results in large finite-basis errors in the PCF near $r=0$.

To enforce the Kimball cusp condition, we replace the short-range region of the PCF with a function that has the appropriate behavior. A plane-wave cutoff energy of $E_{\text {cut }}$ implies that only $\mathbf{G}$ vectors with $G \leq \sqrt{2 E_{\text {cut }}}$ are used to describe the orbitals and hence that variations on a length scale less than $r_{\text {cusp }} \equiv 2 \pi / \sqrt{2 E_{\text {cut }}}$ cannot be described accurately. So we discard the region of the PCF with $r<r_{\text {cusp }}$ and replace it with $\exp \left(c_{0}-r+c_{2} r^{2}+c_{3} r^{3}\right)$, where $c_{0}, c_{2}$, and $c_{3}$ are determined by matching the value, derivative, and second derivative of $g(r)$ at $r=r_{\text {cusp. }}$.

\section{APPENDIX C: LOW-DENSITY LIMITING BEHAVIOR OF THE ELECTRON-POSITRON CONTACT PCF}

For an isolated positronium atom, the normalized wave function is $\psi(r)=\exp (-r / 2) / \sqrt{8 \pi}$. The probability density that the electron and positron coincide is $|\psi(0)|^{2}=1 /(8 \pi)$.

Consider a positron immersed in a HEG at low density. Suppose the system resembles a single positronium atom in a very dilute electron gas in each primitive cell. The density of the center of mass of the positronium atoms is $1 / V_{p}$. Hence the pair density for both the electron and the positron being at $\mathbf{r}$ is $\rho_{2 p}(\mathbf{r}, \mathbf{r}) \approx 1 /\left(8 \pi V_{p}\right)$. The electron and positron densities are $\rho_{1}(\mathbf{r})=3 /\left(4 \pi r_{s}^{3}\right)$ and $\rho_{p}(\mathbf{r})=1 / V_{p}$, respectively. So the contact PCF is $g(0) \approx r_{s}^{3} / 6$.

Now let us make the more realistic assumption that the low-density limit resembles an isolated $\mathrm{Ps}^{-}$ion. For an isolated $\mathrm{Ps}^{-}$ion the electron-positron contact probability density is 0.020733198005108 a.u. (Ref. 24) So in the low-density limit $g(0) \approx 0.1736940334353862647 r_{s}^{3}$.

\footnotetext{
*Present address: University College London, Gower Street, London WC1E 6BT, United Kingdom.

${ }^{1}$ G. D. Mahan, Phys. Rev. 153, 882 (1967).

${ }^{2}$ P. Hohenberg and W. Kohn, Phys. Rev. 136, B864 (1964).
}

\footnotetext{
${ }^{3}$ W. Kohn and L. J. Sham, Phys. Rev. 140, A1133 (1965).

${ }^{4}$ E. P. Gross, Nuovo Cimento 20, 454 (1961).

${ }^{5}$ L. P. Pitaevskii, Sov. Phys. JETP 13, 451 (1961).

${ }^{6}$ R. Krause-Rehberg and H. S. Leipner, Positron Annihilation in
} 
Semiconductors (Springer-Verlag, Berlin, 1999).

${ }^{7}$ E. Boroński and R. M. Nieminen, Phys. Rev. B 34, 3820 (1986).

${ }^{8}$ M. J. Puska and R. M. Nieminen, Rev. Mod. Phys. 66, 841 (1994).

${ }^{9}$ C. H. Leung, M. J. Stott, and C. O. Almbladh, Phys. Lett. 57A, 26 (1976).

${ }^{10}$ G. Rajagopal, R. J. Needs, S. Kenny, W. M. C. Foulkes, and A. James, Phys. Rev. Lett. 73, 1959 (1994).

${ }^{11}$ G. Rajagopal, R. J. Needs, A. James, S. D. Kenny, and W. M. C. Foulkes, Phys. Rev. B 51, 10591 (1995).

${ }^{12}$ S. A. Bonev and N. W. Ashcroft, Phys. Rev. B 64, 224112 (2001).

${ }^{13}$ S. J. Clark, M. D. Segall, C. J. Pickard, P. J. Hasnip, M. J. Probert, K. Refson, and M. C. Payne, Z. Kristallogr. 220, 567 (2005).

${ }^{14}$ G. P. Kerker, Phys. Rev. B 23, 3082 (1981).

${ }^{15}$ J. Arponen, J. Phys. C 11, L739 (1978).

${ }^{16}$ P. O. Löwdin, Phys. Rev. 97, 1474 (1955).

${ }^{17}$ H. J. Monkhorst and J. D. Pack, Phys. Rev. B 13, 5188 (1976).

${ }^{18}$ J. Sherman and W. J. Morrison, Ann. Math. Stat. 21, 124 (1950).

${ }^{19}$ S. Kahana, Phys. Rev. 129, 1622 (1963).

${ }^{20}$ Although the finite-system PCF $g_{N}^{\uparrow}(\mathbf{r})$ must satisfy $\int\left[g_{N}^{\uparrow}(\mathbf{r})\right.$ $-1] d \mathbf{r}=0$, the infinite-system PCF does not necessarily satisfy this normalization condition. In fact, the infinite-system electron-positron PCF $g_{\infty}^{\uparrow}(\mathbf{r})=\lim _{N \rightarrow \infty} g_{N}^{\uparrow}(\mathbf{r})$ satisfies $\int\left[g_{\infty}^{\uparrow}(\mathbf{r})\right.$ $-1] d \mathbf{r} \approx 1$. The reason for this behavior is that in a finite simulation cell containing a single positron, the PCF goes to
$(N-1) / N$ far from the positron, because a single electron is bound to the positron. $(N-1) / N$ tends to 1 as $N \rightarrow \infty$, however, so that the "missing" electron is no longer accounted for in this limit.

${ }^{21}$ L. Mitáš, E. L. Shirley, and D. M. Ceperley, J. Chem. Phys. 95, 3467 (1991).

${ }^{22}$ J. P. Perdew, K. Burke, and M. Ernzerhof, Phys. Rev. Lett. 77, 3865 (1996).

${ }^{23}$ J. C. Kimball, Phys. Rev. A 7, 1648 (1973).

${ }^{24}$ A. M. Frolov, Phys. Lett. A 342, 430 (2005).

${ }^{25}$ J. Arponen and E. Pajanne, Ann. Phys. 121, 343 (1979).

${ }^{26}$ L. J. Lantto, Phys. Rev. B 36, 5160 (1987).

${ }^{27}$ E. Boroński and H. Stachowiak, Phys. Rev. B 57, 6215 (1998).

${ }^{28}$ G. Ortiz, Ph.D. thesis, Swiss Federal Institute of Technology, 1992.

${ }^{29}$ L. Fraser, Ph.D. thesis, Imperial College, 1995.

${ }^{30}$ H. Stachowiak, Phys. Rev. B 41, 12522 (1990).

${ }^{31}$ G. F. Giuliani and G. Vignale, Quantum Theory of the Electron Liquid (Cambridge University Press, Cambridge, 2005).

${ }^{32}$ C. O. Almbladh, U. von Barth, Z. D. Popovic, and M. J. Stott, Phys. Rev. B 14, 2250 (1976).

${ }^{33}$ H. Stachowiak and J. Lach, Phys. Rev. B 48, 9828 (1993).

${ }^{34}$ V. Apaja, S. Denk, and E. Krotscheck, Phys. Rev. B 68, 195118 (2003).

${ }^{35}$ T. Kato, Commun. Pure Appl. Math. 10, 151 (1957); R. T. Pack and W. B. Brown, J. Chem. Phys. 45, 556 (1966). 